H. Umemura

Nagoya Math. J.

Vol. 117 (1990), 125-171

\title{
SECOND PROOF OF THE IRREDUCIBILITY OF THE FIRST DIFFERENTIAL EQUATION OF PAINLEVÉ
}

\author{
HIROSHI UMEMURA
}

In our paper [U2], we proved the irreducibility of the first differential equation $y^{\prime \prime}=6 y^{2}+x$ of Painlevé. In that paper we explained the origin of the problem and the importance of giving a rigorous proof. We can say that our method in [U2] is algebraic and finite dimensional in contrast to a prediction of Painleve who expected a proof depending on the infinite dimensional differential Galois theory. Even nowadays the latter remains to be established. It seems that Painlevé needed an armament with the general theory (the infinite dimensional differential Galois theory) in the controversy with $R$. Liouville on the mathematical foundation of the proof of the irreducibility of the first differential equation (1902-03). Thus he forgot his earlier idea of proving the irreducibility, which is simple and natural and found in the twenty-first lecture of Leçons de Stockholm given in 1895 (von. 1 [P]): a differential equation $y^{\prime \prime}=R\left(x, y, y^{\prime}\right)$ (here $R\left(x, y, y^{\prime}\right)$ is a rational function of $x, y, y^{\prime}$ with coefficients in $\mathbb{C}$ ) free from moving critical points is irreducible if and only if the general solution $y\left(x_{0} ; y_{0}, y_{0}^{\prime} ; x\right)$ (taking the initial condition $y_{0}, y_{0}^{\prime}$ at $x_{0}$ ) is an essentially transcendental function of $\left(y_{0}, y_{0}^{\prime}\right)$. The main result of this paper is a second proof of the irreducibility of $y^{\prime \prime}=6 y^{2}+x$ based on this idea of Painlevé ( $\S 3$, Theorem (68)). The second proof is analytic as the transcendental correspondence is involved. It looks more indirect than the first proof given in [U2] but it has an advantage. In [U1] we had to make the definition of being irreducible precise or equivalently we had to make the permissble operations clear. Essentially they are the solution of linear differential equations and the substitution in Abelian functions (see $\S 1$ ). These operations are related with algebraic groups. But in [U2] we proved a better irreducible theorem: impossibility of solving the differential equation $y^{\prime \prime}=6 y^{2}+x$ by the above 2 operations combined with the solution of first order algebraic differential equations. As we explained above, the first 2 operations are group theoretic but we

Received January 11, 1989. 
do not know the nature of the latter operation. We prove that if the second order differential equation in question is free from moving branch points and moving transcendental singular points and if it is solvable by these 3 operations, then it is solvable by the first 2 group theoretic operations (Theorem (50)). Therefore the irreducibility theorem is understood in a completely group theoretic way.

Here is the content of each section. In $\S 1$, we notice the equivalence of the 2 definitions of the rational dependence on the initial conditions. The first one is due to the classical authors and adopted in our paper [U1]. The second one is an algebraic definition due to Nishioka. The same remark is done for the definition of algebraic dependence. Some complementary remarks on the permissible operations are also done (Lemmas (1.1) and (1.2)).

In $\S 2$, we clear away an ambiguity of our paper [U1] (Corollary (4.6) and Theorem (3.21) in [U1]): if the general solution of an algebraic differential equation depends rationally on the initial conditions, then it is solvable by permissible operations (Theorems (29) and (30)). This result is extended to algebraic differential equations whose general solution depends algebraically on the initial conditions (Theorem (31)). Our works were done independently of Nishioka. But his papers [N2] and [N3] contributed very much to the simplification of arguments. Theorem (23) is due to Nishioka [N3]. We prove Theorem (29) by the same method as in [N3] which with Theorem (30) clears off the ambiguity. Theorem (42) and its Corollary were implicite in the classics and Theorem (42) was first formulated by Nishioka [N2]. Theorem (42) not only plays a substantial role in the proof of other results in $\S 2$ and $\S 3$ but it has also theoretical importance. We simplyfied considerably the arguments of the papers [N2] and [N3]. We are conscious of the following 2 points which seem to be new in this domain though they are now routine in algebraic geometry: (1) a differential equation or a differential field extension $L \supset K$ is characterized by its associated functor (or study not only the extension $L \supset K$ itself but also all the base changes); (2) what is canonical descends and is defined over the smaller base field.

In $\S 3$, we prove a criterion for a differential equation of the second order to be irreducible (Theorem (50)) and apply the Theorem (50) to the first differential equation $y^{\prime \prime}=6 y^{2}+x$.

Thus we put a substantial part of the works of Painlevé on the 
reducibility and the irreducibility of algebraic differential equations of the second order in a definite form except for the following important question. Now we have two proofs of the irreducibility of the first differential equation. Is it possible to give the third proof depending on the infinite dimensional differential Galois theory? The difficulty of establishing the infinite dimensional differential Galois theory is widely recognized. We analyzed however the works of the classical authors and believe that the third proof is in our shooting range.

\section{§1. Differential equations whose general solutions depend rationally or algebraically on the initial conditions}

Let us first settle our notations. An ordinary differential field $(K, \delta)$ consists of, by definition, a field $K$ and a derivation $\delta: K \rightarrow K ; \delta(a+b)$ $=\delta a+\delta b, \delta(a b)=(\delta a) b+a(\delta b)$ for $a, b \in K$. As we are concerned only with ordinary differential fields, we call them simply differential fields. Often we do not make the derivation precise and say a differential field $K$. We assume that the characteristic of the field $K$ is equal to 0 . An element $c \in K$ with $\delta c=0$ is called a constant. The set of the constants of $K$ forms a subfield which we denote by $C_{K}$. A differential field extension $\left(K_{1}, \delta_{1}\right)$ of $(K, \delta)$ is a field extension $K_{1}$ of $K$ such that the derivation $\delta_{1}$ coincides with $\delta$ on $K$. A differential polynomial ring $K\{Y\}$ (of one variable) over a differential field $(K, \delta)$ is a polynomial ring $K\left[Y_{0}, Y_{1}, Y_{2}\right.$, ...] of infinite variables $Y_{i}(i \in N)$, the derivation $\delta$ on the subring $K$ of $K\{Y\}$ being extended on $K\{Y\}$ by $\delta\left(Y_{i}\right)=Y_{i+1}$ for $i \geq 0$. Let $L \supset K$ be a differential field extension and $y \in L$. We denote by $K\langle y\rangle$ (resp. $K\{y\}$ ) the differential field (resp. ring) generated by $y$ over $K$. Let $\left(L_{1}, \delta_{1}\right)$ and $\left(L_{2}, \delta_{2}\right)$ be two differential field extensions of $K$. Then $L_{1} \otimes_{K} L_{2}$ is a differential ring by defining a derivation $\delta$ by $\delta(a \otimes b)=(\delta a) \otimes b+a \otimes \delta b$ for $a \in L_{1}, b \in L_{2}$. The quotient field of an integral domain $A$ will be denoted by $Q(A)$. For example if the field extension $L_{1} \supset K$ is regular, we can speak of a differential field $Q\left(L_{1} \otimes_{K} L_{2}\right)$. The differential field of the meromorphic functions over a domain $D \subset \mathbb{C}$ will be denoted by $K(D)$.

In our papers [U1] and [U2], we introduced the permissible operations of constructing new functions from a set of known functions. We work in a differential field of the meromorphic functions over a domain $D$ of $\mathbb{C}$. Let us recall the permissible operations.

(O) If $f(x)$ is a known function, then the derived function $f^{\prime}(x)$ is a 
new known function.

(P1) If $f$ and $g$ are known functions, then the sum $f+g$, the difference $f-g$ and the product $f g$ are new known functions. Moreover if $g \neq 0$, then the quotient $f / g$ is also a new known function.

(P2) Let $a_{1}, a_{2}, \cdots, a_{n}$ be $n$ known functions. Then an algebroid function $f$ or a solution $f$ of an algebraic equation

$$
f^{n}+a_{1} f^{n-1}+a_{2} f^{n-2}+\cdots+a_{n}=0
$$

is a new known function.

(P3) If $f$ is a known function, then the quadrature $\int f d x$ is a new known function.

(P4) Let $a_{1}, a_{2}, \cdots, a_{n}$ be $n$ known functions. Then any solution $f$ of a linear differential equation

$$
\left((d / d x)^{n}+a_{1}(d / d x)^{n-1}+\cdots+a_{n}\right) f=0
$$

is a new known function.

(P5) Let $\Gamma \subset \mathbb{C}^{n}$ be a lattices such that $\mathbb{C}^{n} / \Gamma$ is an abelian variety. Let $f_{1}, f_{2}, \cdots, f_{n}$ be known holomorphic functions. We denote by $F$ a holomorphic map $D \rightarrow \mathbb{C}^{n} x \mapsto\left(f_{1}(x), f_{2}(x), \cdots, f_{n}(x)\right)$. Then $\varphi \circ p \circ F$ is a new known function for any meromorphic function $\varphi$ on the abelian variety $\mathbb{C}^{n} / \Gamma$. Here we have to avoid the constant function taking the value infinity.

For a meromorphic function on a domain $D$, we identify it with its restriction on a subdomain of $D$ since we are interested in the structure of the field extensions. In other words though we do not mention it explicitly, the restriction of a known meromorphic function on to a subdomain is a new known function.

We proved in [U1] that these permissible operations are related with the algebraic group. However we introduced another permissible operation (P6) in [U2], which breaks the peaceful world of algebraic groups.

(P6) Let $F\left(y, y^{\prime}\right)=0$ be an algebraic differential equation with known coefficients. Then any solution $f$ of $F\left(y, y^{\prime}\right)=0$ is a new known function.

In [U1], we proved the permissible operations are not independent. For example we showed that a combination of $(\mathrm{O}),(\mathrm{P} 1)$ and $(\mathrm{P} 4)$ gives (P2). The proof given there depends on an integral representation of a 
root of an algebraic equation. We give here another proof. The advantage of the new proof is that it is independent of the topology of $\mathbb{C}$.

LEMma (1.1). The permissible operation (P2) is a finite iteration of (O), (P1) and (P4).

Proof. Let $S$ be a starting set of the known functions. Let $K$ be a differential field generated by $S$. Any element of $K$ is known from $S$ by a finite iteration of the permissible operations (O) and (P1). Let $a_{1}$, $a_{2}, \cdots, a_{n} \in S$ and $f$ is a meromorphic solution of an algebraic equation

$$
f^{n}+a_{1} f^{n-1}+\cdots+a_{n}=0
$$

which is irreducible over $K$. Then $K(f)$ is a differential field and $(K(f): K)=n$. Therefore $f, f^{\prime}, f^{(2)}, \cdots, f^{(n)}$ are linearly dependent over $K$. Hence there exist $b_{0}, b_{1}, b_{2}, \cdots, b_{n} \in K$ such that $b_{0} f^{(n)}+b_{1} f^{(n-1)}+\cdots$ $+b_{n} f=0$. Therefore $f$ is a new known functions from the $b_{i}$ 's by (P1), (P4) and hence from the set $S$ by a finite iteration of the permissible operations (O), (P1) and (P4).

We introduced classical functions (Definition (2.27) in [U1]). They are meromorphic functions obtained from the set $\mathbb{C}$ of the constant functions by finite iterations of permissible operations (O), (P1), (P2), , , (P5). Some of our colleagues pointed out that the composition of two classical functions are classical.

Lemma (1.2). Let $f, g$ be two classical functions. Then the composition $f \circ g$ is a classical function.

Proof. We prove the lemma by induction on the number of iteration used to get the function $f$. The lemma is true when $f$ is a constant function. Let us assume that the lemma is true when the function $f$ is obtained from the set $\mathbb{C}$ of the constant functions by at most $i$-time iteration. Let $K_{i}$ be a set of functions obtained from $\mathbb{C}$ by at most $i$-time iterations. Let $f$ be obtained from $\mathbb{C}$ by an $(i+1)$-iteration.

Case (0). If there exists $F \in K_{i}$ such that $f=F^{\prime}$, then $F \circ g$ is classical by induction assumption. Hence $(F \circ g)^{\prime}$ is classical. Since we have $(F \circ g)^{\prime}=\left(F^{\prime} \circ g\right) g^{\prime}$ and since $g^{\prime}$ is classical, by $(\mathrm{P} 1)\left(F^{\prime} \circ g\right)=(F \circ g)^{\prime}\left(g^{\prime}\right)^{-1}$ is classical if $g^{\prime} \neq 0$. If $g^{\prime}=0$, then $g$ is constant and $f \circ g$ is also constant and hence classical.

Case (P1). If there exist $F, G \in K_{i}$ such that $f=F+G$ (or $F-G$, $F G, F / G)$, then $f \circ g=(F \circ g)+(G \circ g)$ is classical by induction assumption 
and (P1). The substraction, the product and the quotient are treated similarly.

Case (P4). If there exist $a_{1}, a_{2}, \cdots, a_{n} \in K_{i}$ such that

$$
f^{(n)}+a_{1} f^{(n-1)}+\cdots+a_{n} f=0,
$$

then we get

$$
\left(f^{(n)}+a_{1} f^{(n-1)}+\cdots+a_{n} f\right) \circ g=0 .
$$

We may assume that $g$ is not constant. We have $(f \circ g)^{\prime}=\left(f^{\prime} \circ g\right) g^{\prime}$ and $(f \circ g)^{\prime \prime}=\left(f^{\prime \prime} \circ g\right)\left(g^{\prime}\right)^{2}+\left(f^{\prime} \circ g\right) g^{\prime \prime}$ and hence

$$
f^{\prime \prime} \circ g=\left(g^{\prime}\right)^{-2}(f \circ g)^{\prime \prime}-\left(f^{\prime} \circ g\right) g^{\prime \prime}\left(g^{\prime}\right)^{-2}=\left(g^{\prime}\right)^{-2}(f \circ g)^{\prime \prime}-(f \circ g)^{\prime} g^{\prime \prime}\left(g^{\prime}\right)^{-3} \text {. }
$$

Since the similar relation holds for $(f \circ g)^{(j)}(j \geq 2)$, $(*)$ gives us a non trivial homogeneous linear differential equation of order $n$ satisfied by $(f \circ g)$ with classical coefficients. Therefore $f \circ g$ is classical.

Case (P5). Let $a_{1}, a_{2}, \cdots, a_{n} \in K_{i}$ and $\Gamma \subset \mathbb{C}^{n}$ be a lattice such that $\mathbb{C}^{n} / \Gamma$ is an abelian variety. Let $F: D \rightarrow \mathbb{C}^{n}, x \rightarrow\left(a_{1}, a_{2}, \cdots, a_{n}\right)$ and $\varphi$ an abelian function with respect to $\Gamma$ ( $\varphi$ is a meromorphic function over $\mathbb{C}^{n}$ invariant by $\Gamma$ ) such that $f=\varphi \circ F$. Then since $f \circ g=(\varphi \circ F) \circ g=$ $\varphi \circ(F \circ g)$ and since $a_{i} \circ g$ is classical by induction hypothesis, $f \circ g$ is classical too.

Since we proved in [U1] that the operations (P2) and (P3) are particular combinations of $(\mathrm{O}),(\mathrm{P} 1),(\mathrm{P} 2), \cdots,(\mathrm{P} 5)$, the lemma is proved.

We prove in [U1] the permissible operations $(\mathrm{O}),(\mathrm{P} 1),(\mathrm{P} 2), \cdots,(\mathrm{P} 5)$ are dominated by the algebraic groups defined over $\mathbb{C}$. Let $D \subset \mathbb{C}$ be a domain and $G$ an algebraic group defined over $\mathbb{C}$. For a holomorphic map $F: D \rightarrow G$, we have defined in [U1] a holomorphic map $\partial F: D \rightarrow \operatorname{Lie} G$, where Lie $G$ is the Lie algebra of $G$. Let us recall briefly the definition of $\partial F$. The holomorphic curve $F: D \rightarrow G$ defines a tangent vector $X_{F(x)}$ at $F(x) \in G$ along $D$. We translate it to a tangent vector at $1 \in G$ by the right translation $R_{F^{(x)-1}}$. $\partial F: D \rightarrow \operatorname{Lie} G$ is defined by $(\partial F)(x)=R_{F(x)-1 *} X_{F(x)}$. We introduced the operation (Q).

(Q) Let $f_{1}, f_{2}, \cdots, f_{d}$ be holomorphic known functions over $D$. Let $F: D \rightarrow G$ be a holomorphic map such that with a suitable base of Lie $G$, $\partial F: D \rightarrow$ Lie $G \simeq \mathbb{C}^{d}$ is given by $x \simeq\left(f_{1}(x), f_{2}(x), \cdots, f_{d}(x)\right)$. Then $\varphi \circ F$ is a new known function for any rational function $\varphi \in \mathbb{C}(G)$ on $G$.

We proved 
Theorem (2). Let $S$ be a starting set of known meromorphic functions. $A$ meromorphic function is obtained from $S$ by a finite iteration of the permissible operations $(\mathrm{O}),(\mathrm{P} 1),(\mathrm{P} 2), \cdots,(\mathrm{P} 5)$ if and only if it is obtained from $S$ by a finite iteration of $(\mathrm{O})$ and $(\mathrm{Q})$.

In the study of the structure of algebraic differential equations, it is often more advantageous to work in an abstract setting than in concrete analytic terms. Let $K$ be a differential field and $C_{K}$ be the field of the constants of $K$. We defined in [U1] for a $K$-valued point $v$ : Spec $K$ $\rightarrow G$ (or more precisely for the corresponding $K$-rational point $v$ : Spec $K$ $\rightarrow G \otimes_{C_{K}} K$ ) over $C_{K}$ a logarithmic derivative $l \delta\left(v^{\prime}\right) \in K \otimes_{C_{K}}$ Lie $G$ (see also Kolchin [K2]). We can characterize the permissible operations in a group theoretic way.

THEOREM (3). Let $M$ be a differential field of known meromorphic functions on a domain $D$ and $L$ be the differential field of all the meromorphic functions on $D$. We assume that $M$ contains the field $\mathbb{C}$ of the constant functions on $D: \mathbb{C} \subset M \subset L$. To apply the operations (O), (Q), to $M$ is equivalent to allowing the following extension of the differential field $M$. Let $v$ : Spec $L \rightarrow G_{M}=G \otimes_{\mathrm{c}} M$ be an L-valued point over $M$. The corresponding L-rational point Spec $L \rightarrow G_{L}=G \otimes_{\mathrm{c}} L$ is denoted by $v^{\prime}$. If $l \delta\left(v^{\prime}\right) \in$ Lie $G \otimes_{\mathrm{C}} L$ lies in Lie $G \otimes_{\mathbb{C}} M$, then the image $O_{G_{M}}, v / \mathfrak{m}_{v} \subset L$ is a differential field of newly known functions. Moreover this differential field is finite type over $M$ as an abstract field extension.

We are interested in the meromorphic solutions but it is convenient to work in bigger differential fields. A natural generalization of the operation $(\mathrm{Q})$ is as follows.

Definition (4). Let $M \subset L$ be an extension of differential field. Let $C=C_{M}$ be the field of the constants of $M$ and $G$ an algebraic group defined over $C$. Let $v$ : Spec $L \rightarrow G_{M}=G \otimes_{C} M$ be an $L$-valued point. The corresponding $L$-rational point will be denoted by $v^{\prime}: \operatorname{Spec} L \rightarrow G_{L}$. If $l \delta\left(v^{\prime}\right) \in$ Lie $G_{L}=$ Lie $G \otimes_{C} L$ lies in Lie $G \otimes_{C} M$, then the image of $O_{G_{M}, v} / \mathrm{m}_{v}$ in $L$ is called a $G$-primitive extension of $M$.

Notice that the extension is of finite type over $M$ and is a differential field (cf. [U2] Remark 18.3 and [K2]).

The following Proposition is crucial ([U2], Corollary 18.2 and [K2]).

Proposition (5). Let $L \supset K$ be a G-primitive extension. If the field 
extension is regular, then the differential field $Q\left(L \otimes_{K} L\right)$ is generated over $i_{1}(L)\left(\right.$ resp. $\left.i_{2}(L)\right)$ by constants, where $i_{1}\left(\right.$ resp. $\left.i_{2}\right): L \rightarrow L \otimes_{K} L \subset Q\left(L \otimes_{K} L\right)$ is defined by $a \rightarrow a \otimes 1$ (resp. $1 \otimes a)$.

Let $D \subset \mathbb{C}$ be a domain and $x$ be the parameter on $\mathbb{C}$. We denote by $K(D)$ the field of the meromorphic function on $D$. Let $R$ be a $\mathbb{C}$ algebra of holomorphic functions on $D$ and $F\left(Y_{0}, Y_{1}, Y_{2}, \cdots, Y_{n}\right) \in R\left[Y_{0}, Y_{1}\right.$, $\left.Y_{2}, \cdots, Y_{n}\right]$ a polynomial. We write often $F\left(x ; Y_{0}, Y_{1}, Y_{2}, \cdots, Y_{n}\right)$ to show that the coefficients of $F(Y)$ are functions of $x$. We have a map $i: D \rightarrow$ Spec $R$ by putting $i(x)=\{f \in R \mid f(x)=0\}$. If $R$ is of finite type over $\mathbb{C}, i$ induces a morphism $i: D \rightarrow(\operatorname{Spec} R)^{\text {an }}$ of analytic spaces. Let $\mathscr{X}$ be an algebraic subvariety defined by $F$ in $A^{n+1} \otimes_{\mathrm{c}} R=A^{n+1} \times \operatorname{Spec} R: \mathscr{X}=$ $V(F) \subset A_{R}^{n+1}=\operatorname{Spec} R\left[Y_{0}, Y_{1}, \cdots, Y_{n}\right]$. Similarly we define $X=\{(x, z) \in D$ $\left.\times A_{\mathrm{c}}^{n+1} \mid F(x, z)=0\right\}$ which is an analytic space. We have a diagram

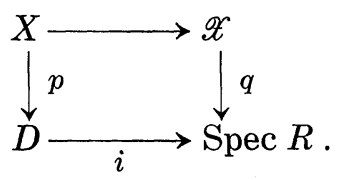

If $R$ is of finite type over $\mathbb{C}$, we proved that the diagram

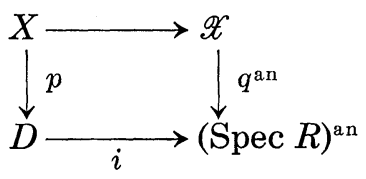

is cartesian in the category of the analytic spaces ([U1] $\S 3$ ). We denote by $X_{\xi}$ the fibre $p^{-1}(\xi)$ over a point $\xi \in D$ and we use the similar notation for $q$.

We are interested in an algebraic differential equation

(6) $F\left(x ; y, y^{\prime}, \cdots, y^{(n)}\right)=0$.

In general in the theory of algebraic differential equation, we assume, without expressing it, that the polynomial $F\left(Y_{0}, Y_{1}, Y_{2}, \cdots, Y_{n}\right)$ is absolutely irreducible. Let $x_{0} \in D$ be a general point so that the coefficients of the polynomial $F$ are regular at $x_{0}$, the algebraic variety $X_{0}=X_{x_{0}}=$ $\left\{\left(z_{0}, z_{1}, \cdots, z_{n}\right) \in \mathbb{C}^{n+1} \mid F\left(x_{0} ; z_{0}, z_{1}, \cdots, z_{n}\right)=0\right\}$ is irreducible ([U1]) and so that the Zariski open set $U=\left\{z \in X_{0} \mid \partial F / \partial Y_{n}\left(x_{0} ; z\right) \neq 0\right\} \subset X_{0}$ is not empty. For $z \in U$, there exists a solution $y\left(x_{0} ; z ; x\right)$ of (6) taking the initial value $z$ at $x_{0} ; y^{(i)}\left(x_{0} ; z_{0}, z_{1}, \cdots, z_{n} ; x_{0}\right)=z_{i}$ for $0 \leq i \leq n$. The function $y\left(x_{0} ; z ; x\right)$ is holomorphic both in $x$ and $z=\left(z_{0}, z_{1}, \cdots, z_{n}\right)$. To be precise for any 
point $P \in U$, there exist an open neighbourhood $V \subset U$ of $P$, an open neighbourhood $W \subset D$ of $x_{0}$ and a holomorphic function $y\left(x_{0} ; z ; x\right)$ on $V \times W$ of $(z, x)$ which satisfies the differential equation and takes the initial value $z \in V$ at $x_{0}$. As we see in Painlevé's Leçons de Stockholm $[\mathrm{P}]$, the nature of the solution $y\left(x_{0} ; z ; x\right)$ as the function of the initial conditions $z$ is closely related with the degree of the difficulty of solving the differential equation (6). For example we proved in [U1] that if $y\left(x_{0}: z ; x\right)$ depends rationally on the initial conditions $z$, we can solve the equation (6) starting from the ring $R$ of the known functions by a combination of linear differential equations and abelian functions (cf. [U1] and $\S 2$, Theorem (29) and Theorem (30)). We notice that this result contains as a special case a theorem of Poincaré.

Theorem (Poincaré). Let $F\left(x ; y, y^{\prime}\right)=0$ be an algebraic differential equation without moving critical points. Then we can solve it starting from the known functions by linear differential equations and elliptic functions (the coefficients of $F$ being known functions).

A precise assertion will be given under more general assumptions (§ 2, Theorems (28), (30) and (31)).

Let us recall and examine the definition that the general solution $y\left(x_{0} ; z ; x\right)$ depends rationally on the initial conditions $z$ since we are going to generalize it so that we can prove an irreduciblity theorem by studying the general solution as a function of the initial conditions.

Definition (7). We say that the general solution $y\left(x_{0} ; z_{0}, z_{1}, \cdots, z_{n} ; x\right)$ of (6) depends rationally on the initial conditions if there exist a general point $x_{0}$ (fixed once for all), a non-empty open set $V \subset U \subset X_{0}$ for the usual topology, a domain $x_{0} \in D^{\prime} \subset D$ and a polynomials $C_{i}\left(x ; Y_{0}, Y_{1}, \cdots\right.$, $\left.Y_{n}\right), D_{i}\left(x ; Y_{0}, Y_{1}, \cdots, Y_{n}\right)$ with coefficients in a ring of holomorphic functions on $D^{\prime}$ such that (1) $y^{(i)}\left(x_{0} ; z ; x\right)$ is holomorphic for $(z, x) \in V \times D^{\prime}$ for $0 \leq i \leq n$, (2) $D_{i}\left(x_{0} ; z_{0}, z_{1}, \cdots, z_{n}\right) \neq 0$ for $(z, x) \in V \times D^{\prime}$. (3) $y^{(i)}\left(x_{0} ; z_{0}\right.$, $\left.z_{1}, \cdots, z_{n} ; x\right)=C_{i} / D_{i}\left(x ; z_{0}, z_{1}, \cdots, z_{n}\right)$ for $(z, x) \in V \times D^{\prime}$.

As we see in Proposition (16) below, the geometric meaning of Definition (7) is that replacing $D^{\prime}$ by a smaller domain if necessary, the map $z \rightarrow\left(y\left(x_{0} ; z ; x_{1}\right), y^{\prime}\left(x_{0} ; z ; x_{1}\right), \cdots, y^{(n)}\left(x_{0} ; z ; x_{1}\right)\right)$ defines a birational correspondence between the algebraic varieties $X_{0}$ and $X_{1}=\left\{\left(z_{0}, z_{1}, \cdots, z_{n}\right)\right.$ $\left.\in \mathbb{C}^{n+1} \mid F\left(x_{1} ; z_{0}, z_{1}, \cdots, z_{n}\right)=0\right\}$. 
Remark (8). In Definition (7), by a general point $x_{0} \in D$, we mean a point $x_{0} \in D$ such that the function $\partial F / \partial Y_{n}\left(x_{0} ; Y\right)$ on $X_{0}$ is not constantly equal to 0 and such that $p^{-1}\left(x_{0}\right)$ is irreducible and reduced. These conditions are satisfied for any point of a dense open set of $D$. We see later the definition is independent of the reference point $x_{0}$. Namely if there exists a point satisfying the conditions of the definition, then the conditions of the definition are satisfied by any point of a dense open subset of $D$.

Notice we proved the following result in [U1], which we do not use in the sequel. If the ring $R$ is of finite type over $\mathbb{C}$, Definition (7) implies that

(9) there exist a $\mathbb{C}$-algebra $R^{\prime}$ consisting of holomorphic functions on $D^{\prime}$ and containing $R$ and of finite type over $\mathbb{C}$ and an algebraic variety $\mathscr{Z} \subset X_{0} \times \mathscr{X} \otimes_{R} R^{\prime}=\left(X_{0} \otimes_{C} R^{\prime}\right) \times_{R^{\prime}}\left(\mathscr{X} \otimes_{R} R^{\prime}\right)$ such that (1) $\mathscr{Z}$ defines an $R^{\prime}$-birational correspondence between the algebraic varieties $X_{0} \times \operatorname{Spec} R^{\prime}$ and $\mathscr{X} \times_{\text {Spec } R} \operatorname{Spec} R^{\prime}$ or equivalently at each point $u \in \operatorname{Spec} R^{\prime}$, the fibre $\mathscr{Z}_{u}$ defines a birational correspondence of $X_{0}$ and $\mathscr{X}_{u}$ such that (2) $\left(z, y\left(x_{0} ; z ; x\right), y^{\prime}\left(x_{0} ; z ; x\right), \cdots, y^{(n)}\left(x_{0} ; z ; x\right)\right) \in X_{0} \times \mathscr{X}_{i\left(X_{0}\right)}=\left(\left(X_{0} \times R^{\prime}\right) \times_{R^{\prime}}\right.$ $\left.\left(\mathscr{X} \otimes_{R} R^{\prime}\right)\right)_{i(X)}$ is in $\mathscr{Z}$ for $x \in D^{\prime}$ (see Proposition (16) below).

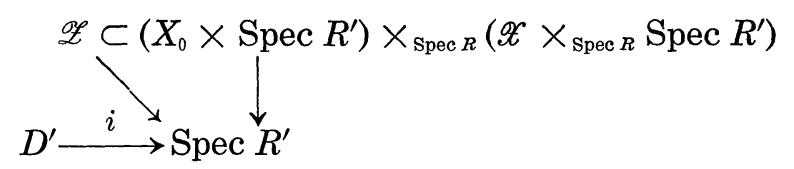

We look for equivalent conditions to Definition (7). Let $z=\left(z_{0}, z_{1}\right.$, $\cdots, z_{n}$ ) be the generic point of $X_{0}$ and $x_{0}$ be as above. We consider $z$ as a variable point in an open set of $U \subset X_{0}$ for the usual topology. We have the following conditions.

(9.1) The general solution of the differential equation (6) depends rationally on the initial conditions.

(9.2) There exist a (fixed general) point $x_{0}$, a non-empty open set $V$ of $X_{x_{0}}$ for the usual toplogy and a domain $x_{0} \in D^{\prime} \subset D$ such that the functions $y\left(x_{0} ; z ; x\right), y^{\prime}\left(x_{0} ; z ; x\right), \cdots, y^{(n)}\left(x_{0} ; z ; x\right)$ in $(z, x)$ are holomorphic on $V \times D^{\prime}$ and such that the field $K\left(D^{\prime}\right)\left(y\left(x_{0} ; z ; x\right), y^{\prime}\left(x_{0} ; z ; x\right), \cdots, y^{(n)}\left(x_{0}\right.\right.$; $z ; x)$ ) is a subfield of the field $K\left(D^{\prime}\right)\left(z_{0}, z_{1}, \cdots, z_{n}\right)$. Here all the functions and fields are considered in the field of meromorphic functions on $V \times$ $D^{\prime}$.

(9.3) There exist a (fixed general) point $x_{0}$, a non-empty open set $V$ 
of $X_{x_{0}}$ for the usual topology and a domain $x_{0} \in D^{\prime} \subset D$ such that the functions $y\left(x_{0} ; z ; x\right) ; y^{\prime}\left(x_{0} ; z ; x\right), \cdots, y^{(n)}\left(x_{0} ; z, x\right)$ in $(z, x)$ are holomorphic on $V \times D^{\prime}$ and such that the field $K\left(D^{\prime}\right)\left(y\left(x_{0} ; z ; x\right), y^{\prime}\left(x_{0} ; z ; x\right), \cdots, y^{\prime}\left(x_{0}\right.\right.$; $z ; x)$ ) coincides with the field $K\left(D^{\prime}\right)\left(z_{0}, z_{1}, \cdots, z_{n}\right)$ the same identification as in the condition (2) being done.

Lemma (10). The conditions (9.1) and (9.2) are equivalent. The condition (9.3) implies the conditions (9.2) and (9.1).

Proof. The equivalence of the conditions (9.1) and (9.2) follows from the definition. The condition (9.3) is stronger than (9.2).

Nishioka introduced the following definition of rational dependence on the initial conditions in an abstract setting.

Definition (11). Let $L \supset K$ be a differential field extension. We assume that the field extension $L \supset K$ is regular and of finite type. We say that the extension $L \supset K$ depends rationally on the initial conditions if there exists a differential field extension $M \supset K$ such that the quotient field $Q\left(L \otimes_{K} M\right)$ which is a differential field extension of $M$, is generated over $M$ by constants (cf. [N3]).

Remark (11.1). It follows from the definition that for any differential field extension $J \supset K$, an extension $L \supset K$ depends rationally on the initial conditions if and only if the extension $Q\left(L \otimes_{K} J\right) \supset J$ depends rationally on the initial conditions. We can say that the notion of the rational dependence on the initial conditions of $L \supset K$ is free from the base change.

Let us see how Definitions (7) and (11) are related. Let us take in Definition (7) as $K$ the differential field generated over $\mathbb{C}$ by $R$. Thus $K$ is a differential subfield of $K(D)$. Unfortunately a differential ideal $\{F\}$ generated by $F\left(Y_{0}, Y_{1}, \cdots, Y_{n}\right)$ in a differential polynomial ring $K\{Y\}$ is not prime. But it determines a differential prime ideal $\mathfrak{P}$ of the generic solution such that the quotient differential field of $K\{Y\} / \Re$ is $K$-isomorphic to $K\left(y\left(x_{0} ; z ; x\right), y^{(1)}\left(x_{0} ; z ; x\right), \cdots, y^{(n)}\left(x_{0} ; z ; x\right)\right)$ (see Kolchin [K2], Chap. IV, $\S 6)$. Let $M$ be the differential field $K\left(D^{\prime}\right)$. Then $M$ and $K\left(y\left(x_{0} ; z ; x\right)\right.$, $\left.y^{(1)}\left(x_{0} ; z ; x\right), \cdots, y^{(n)}\left(x_{0} ; z ; x\right)\right)$ are linearly disjoint over $K$. Therefore $M\left(y\left(x_{0} ; z ; x\right), y^{(1)}\left(x_{0} ; z ; x\right), \cdots, y^{(n)}\left(x_{0} ; z ; x\right)\right)$ is isomorphic to the quotient field of $K\left(y\left(x_{0} ; z ; x\right), y^{(1)}\left(x_{0} ; z ; x\right), \cdots, y^{(n)}\left(x_{0} ; z ; x\right)\right) \otimes_{K} M$. It follows from Definition (7) that the field $M\left(y\left(x_{0} ; z ; x\right), y^{(1)}\left(x_{0} ; z ; x\right), \cdots, y^{(n)}\left(x_{0} ; z ; x\right)\right)$ is 
contained in a field $M\left(z_{0}, z_{1}, \cdots, z_{n}\right)$ generated over $M$ by the constants $z_{0}, z_{1}, \cdots, z_{n} ; M\left(y\left(x_{0} ; z ; x\right), y^{(1)}\left(x_{0} ; z ; x\right), \cdots, y^{(n)}\left(x_{0} ; z ; x\right)\right) \subset M\left(z_{0}, z_{1}, \cdots, z_{n}\right)$. Let us put $M_{1}=M\left(y\left(x_{0} ; z ; x\right), y^{(1)}\left(x_{0} ; z ; x\right), \cdots, y^{(n)}\left(x_{0} ; z ; x\right)\right)$ and $M_{2}=$ $M\left(z_{0}, z_{1}, \cdots, z_{n}\right)$. We have a diagram

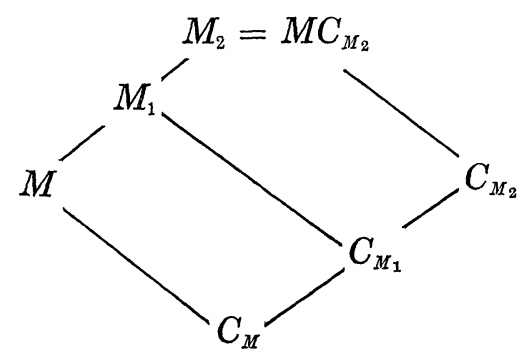

where $C_{M}, C_{M_{1}}$ and $C_{M_{2}}$ denote the field of constants of the differential fields. It follows from the following proposition (due to Kolchin [K2], Chap. II, $\S 1$, Corollary 2 on p. 88) that $M_{1}=M C_{M_{1}}$.

Proposition (12). Let $M \subset N$ be a differential field extension generated over $M$ by constants so that $N=C_{N} M$. Then there is a 1:1 correspondence between the elements of the following two sets.

(1) The set $S$ of the intermediate differential fields $M \subset J \subset N$.

(2) The set $T$ of the intermediate fields $C_{M} \subset D \subset C_{N}$.

Here the map $S \rightarrow T$ is given by $J \cap C_{N}$ for $J \in S$ and the map $T \rightarrow S$ is given by $D M$ for $D \in T$. In particular for any $J \in S$ we have $J=C_{J} M$.

We have seen above that Definition (7) is a special case of Definition (11). Let us now show the converse. Let us denote by $L$ a differential field $K\left(y\left(x_{0}, z ; z\right), y^{(1)}\left(x_{0}, z ; x\right), \cdots, y^{(n)}\left(x_{0} ; z ; x\right)\right)$ which is a regular differential field extension of $K$. We assume that there exists a differential field $M$ such that the quotient field $Q\left(L \otimes_{K} M\right)$ is generated over $M$ by constants. Since $L$ is finitely generated over $K$ and since in the definition only a finite number of elements are involved, we may assume that the differential field $K$ is finitely generated over $\mathbb{Q}$ as a differential field and $M$ is finitely generated over $K$ as a differential field. Hence the differential field $M$ is finitely generated over $\mathbb{Q}$. Therefore by a theorem of Seidenberg [S2] we may assume that there exists a subdomain $D^{\prime} \subset D$ such that $K \subset M \subset K\left(D^{\prime}\right)$. Here we identify the field $K$ of meromorphic functions on $D$ with a subfield of meromorphic functions on $D^{\prime} \subset D$ by restriction. It follows from the proof of [S2] that the 
choice of the domain $D^{\prime}$ is rather free. In fact it is shown in [S2] in the course of the proof that there exists a subset $E^{\prime} \subset D$ such that (1) $D-E^{\prime}$ is a countable union of analytic subsets of $D$ and such that (2) for any point $x_{1} \in D-E^{\prime}$ we can choose a subdomain $D^{\prime}$ containing $x_{1}$ satisfying $K \subset M \subset K\left(D^{\prime}\right)$. We may assume that there exists a non-empty open set $V \subset U \subset X_{0}$ for the usual topology such that the functions $y\left(x_{0} ; z ; x\right), y^{(1)}\left(x_{0} ; z ; x\right), \cdots, y^{(n)}\left(x_{0} ; z ; x\right)$ in $(z, x)$ are regular on $V \times D^{\prime}$. Moreover we may assume that

(13) for $x_{1} \in D^{\prime},\left\{\left(y\left(x_{0} ; z ; x_{1}\right), y^{(1)}\left(x_{0} ; z ; x_{1}\right), \cdots, y^{(n)}\left(x_{0} ; z ; x_{1}\right) \in U \mid z \in V\right\}\right.$ contains an open set of $X_{x_{1}}$ for the usual topology. We consider all the involved fields as subfields of the meromorphic functions on $V \times D^{\prime}$. Then since $K\left(y\left(x_{0} ; z ; x\right), y^{(1)}\left(x_{0} ; z ; x\right), \cdots, y^{(n)}\left(x_{0} ; z ; x\right)\right)$ and $M$ are linearly disjoint over $K, M\left(y\left(x_{0} ; z ; x\right), y^{(1)}\left(x_{0} ; z ; x\right), \cdots, y^{(n)}\left(x_{0} ; z ; x\right)\right)$ is isomorphic to the quotient field of $K\left(y\left(x_{0} ; z ; x\right), y^{(1)}\left(x_{0} ; z ; x\right), \cdots, y^{(n)}\left(x_{0} ; z ; x\right)\right) \otimes_{K} M$ and hence generated over $M$ by constants $u_{1}, u_{2}, \cdots, u_{l}$. Let $M^{\prime}=\mathbb{C}(M) \subset K\left(D^{\prime}\right)$ $\subset K\left(V \times D^{\prime}\right)$ and $L^{\prime}=L\left(M^{\prime}\right)=M^{\prime}\left(y\left(x_{0} ; z ; x\right), y^{(1)}\left(x_{0} ; z ; x\right), \cdots, y^{(n)}\left(x_{0} ; z ; x\right)\right)$. Then $L^{\prime}$ is generated over $M^{\prime}$ by constants $w_{1}, w_{2}, \cdots, w_{m}$ in $L^{\prime}: M^{\prime}\left(y\left(x_{0}\right.\right.$; $\left.z ; x), y^{(1)}\left(x_{0} ; z ; x\right), \cdots, y^{(n)}\left(x_{0} ; z ; x\right)\right)=M^{\prime}\left(w_{1}, w_{2}, \cdots, w_{m}\right)$. Hence there exist polynomials $C_{i}(x ; W), D_{i}(x ; w) \in M^{\prime}\left[W_{1}, W_{2}, \cdots, W_{m}\right], E_{j}(x ; Y), F_{j}(x ; Y) \in$ $M^{\prime}\left[Y, Y_{1}, \cdots, Y_{n}\right] 0 \leq i \leq n, 1 \leq j \leq m$ such that

(i) $y^{(i)}\left(x_{0} ; z ; x\right)=C_{i} / D_{i}\left(x ; w_{1}, w_{2}, \cdots, w_{m}\right)$ for $0 \leq i \leq n$,

(ii) $w_{j}=E_{j} / F_{j}\left(y\left(x_{0} ; z ; x\right), y^{(1)}\left(x_{0} ; z ; x\right), \cdots, y^{(n)}\left(x_{0} ; z ; x\right)\right)$ for $1 \leq j \leq m$.

Let now $x_{0}^{\prime} \in D^{\prime}$ such that $D_{i}\left(x_{0}^{\prime} ; w_{1}, w_{2}, \cdots, w_{m}\right) \neq 0$. Let $\mathscr{W}=$ Spec $C\left[w_{1}, w_{2}, \cdots, w_{m}\right]$. We have a $\mathbb{C}$-rational map $f: \mathscr{W} \cdots \rightarrow X_{x_{0}}, w=$ $\left(w_{1}, w_{2}, \cdots, w_{m}\right) \rightarrow\left(C_{0} / D_{0}\left(x_{0}^{\prime} ; w\right), C_{1} / D_{1}\left(x_{0}^{\prime} ; w\right), \cdots, C_{n} / D_{n}\left(x_{0}^{\prime} ; w\right)\right)$ of the algebraic variety $\mathscr{W}$ to $X_{x_{0}^{\prime}}$. We show that the map $f$ is birational. The rational map is dominant by (13). Let $a, b$ be general points of $\mathscr{W}$. If $C_{0} / D_{0}\left(x_{0}^{\prime} ; a\right)=C_{0} / D_{0}\left(x_{0}^{\prime} ; b\right)$, then $C_{0} / D_{0}(x ; a)$ and $C_{0} / D_{0}(x ; b)$ are solutions of the differential equation (6) taking the same initial conditions and hence $C_{0} / D_{0}(x ; a)=C_{0} / D_{0}(x ; b)$. It follows from (i) and (ii) $a=b$ and the degree of the rational map $f$ is equal to 1 . Namely $f$ is birational; $\mathbb{C}\left(X_{x_{0}}\right) \simeq$ $\mathbb{C}(\mathscr{W})$. We have a diagram

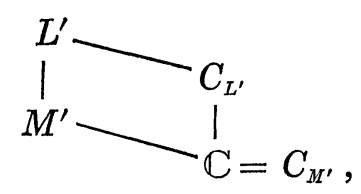


where $C_{L^{\prime}}$ and $C_{M^{\prime}}$ denote the fields of constants of $L^{\prime}$ and $M^{\prime}$. It follows from [K2], Chap. II, $\S 1$, Corollary 1 that $M^{\prime}$ and $C_{L^{\prime}}$ are linearly disjoint over $\mathbb{C}$, hence $M^{\prime}\left(\mathscr{W} \otimes_{\mathbf{c}} M^{\prime}\right) \simeq M^{\prime}\left(w_{1}, w_{2}, \cdots, w_{m}\right)$. Consequently $M^{\prime}\left(X_{x_{0}^{\prime}} \otimes_{\mathbf{C}} M^{\prime}\right) \simeq M^{\prime}\left(\mathscr{W} \otimes_{\mathbf{c}} M^{\prime}\right) \simeq M^{\prime}\left(w_{1}, w_{2}, \cdots, w_{m}\right)=M^{\prime}\left(y\left(x_{0} ; z ; x\right), y\left(x_{0} ;\right.\right.$ $\left.z ; x), \cdots, y^{(n)}\left(x_{0} ; z ; x\right)\right)$. Therefore the condition of Definition (7) is satisfied if we take $x_{0}^{\prime}$ for the reference point. Hence Definitions (7) and (11) are equivalent in the analytic case.

The above argument shows the equivalence of the conditions (9.1) and (9.3). However we notice in the converse, it may happen that we have to choose a new reference point. The choice is rather free as we noticed above.

Proposition (16). The notation being as above. The following conditions are equivalent.

(1) The general solution of the differential equation depends rationally on the initial conditions.

(2) There exists a (general) point $x_{0}$ and a dcmain $x_{0} \in D^{\prime} \subset D$ such that a field $K\left(D^{\prime}\right)\left(y\left(x_{0} ; z ; x\right), y^{\prime}\left(x_{0} ; z ; x\right), \cdots, y^{(n)}\left(x_{0} ; z ; x\right)\right)$ coincides with a subfield $K\left(D^{\prime}\right)\left(z_{0}, z_{1}, \cdots, z_{n}\right)$.

(3) There exist a point $x_{0} \in D$ and a domain $x_{0} \in D^{\prime} \subset D$ such that $\left(z_{0}, z_{1}, \cdots, z_{n}\right) \rightarrow\left(y\left(x_{0} ; z ; x\right), y^{\prime}\left(x_{0}, z ; x\right), \cdots, y^{(n)}\left(x_{0} ; z ; x\right)\right)$ gives $a \quad K\left(D^{\prime}\right)$ birational equivalence of $X_{0} \otimes_{\mathrm{C}} K\left(D^{\prime}\right)$ and $\mathscr{X} \otimes_{R} K\left(D^{\prime}\right)$.

Proof. The conditions (2) and (3) are equivalent by definition. The condition (1) follows from (2) by definition. The above argument shows the condition (1) implies the condition (2).

The following proposition is also a result of the above argument.

Proposition (17). If the general solution of the differential equation depends rationally on the initial conditions, then there is a dense open set $E$ of $D$ such that for any $x_{0} \in E$ and for any point $p \in U \subset X_{x_{0}}$, there exists an open neighbourhood $V \subset U$ of $p$ and a subdomain $x_{0} \in D^{\prime} \subset D$ and polynomials satisfying the condition of Definition (7). Namely the definition that the general solution depends rationally on the initial conditions is free from the reference point.

Proof. Let $\xi \in D$ such that $X_{\xi}$ is reduced and irreducible and such that $\partial F / \partial Y_{n}(\xi ; z)$ is not constantly equal to 0 on $X_{\xi}$. Let $y(\xi ; z ; x)$ be the general solution taking the initial condition $z$ at $\xi$. Let $\xi^{\prime}$ be another 
point satisfying the same condition. Then the extension $K\langle y(\xi ; z ; x)\rangle$ is $K$-isomorphic to $K\left\langle y\left(\xi^{\prime} ; z ; x\right)\right\rangle$. As we have seen above, if the condition of Definition (7) is satisfied, then the condition of Definition (11) is satisfied. When we proved the converse, first we had to realize $M \supset K$ (such that $Q\left(K\langle y(\xi ; z ; x)\rangle \otimes_{K} M\right)$ is generated over $M$ by constants) as a subfield of $K\left(D^{\prime}\right)$ for some sudomain $D^{\prime} \subset D$ (here we notice that $M$ is determined independently from $\xi$ ). As we explained above, this can be done almost freely: except for an enumerative points of $D$, we can find a neighbourhood $D^{\prime} \subset D$ satisfying the required conditions. Next if we choose a point $x_{0} \in D^{\prime}$ such that $D_{i}\left(x_{0}^{\prime} ; w_{1}, w_{2}, \cdots, w_{m}\right) \neq 0$, then the argument above shows that $x_{0}$ can be a reference point of Definition (7). Therefore any point of a non-empty Zariski open set of $D^{\prime}$ can be chosen as a reference point.

A natural generalization of rational dependence on the initial conditions is that the general solution $y\left(x_{0} ; z ; x\right)$ is an algebraic function of the initial conditions $z \in X_{0}$. We have equivalent conditions.

Lemma (18). Notation being as above, the following conditions are equivalent.

(1) There exist a (fixed general) point $x_{0} \in D$ and a domain $x_{0} \in D^{\prime} \subset D$ such that a field $K\left(D^{\prime}\right)\left(z_{0}, z_{1}, \cdots, z_{n}, y\left(x_{0} ; z ; x\right), y^{\prime}\left(x_{0} ; z ; x\right), \cdots, y^{(n)}\left(x_{0} ; z ; x\right)\right)$ is algebraic over $K\left(D^{\prime}\right)\left(z_{0}, z_{1}, \cdots, z_{n}\right)$. Here the involved functions are considered as functions on $D^{\prime} \times V$ for a suitable open set $V$ on $X_{x_{0}}$ for the usual topology as in (9.2). Hence all the fields are subfields of the field of the meromorphic functions on $D^{\prime} \times V$.

(2) There exist a fixed general point $x_{0}$ and a domain $x_{0} \in D^{\prime} \subset D$ such that the field $K\left(D^{\prime}\right)\left(z_{0}, z_{1}, \cdots, z_{n}, y\left(x_{0} ; z ; x\right), y^{\prime}\left(x_{0} ; z ; x\right), \cdots, y^{(n)}\left(x_{0} ; z\right.\right.$; $x)$ ) is algebraic over $K\left(D^{\prime}\right)\left(y\left(x_{0} ; z ; x\right), y^{\prime}\left(x_{0} ; z ; x\right), \cdots, y^{(n)}\left(x_{0} ; z ; x\right)\right)$.

(3) There exist a fixed general point $x_{0}$ and a domain $x_{0} \in D^{\prime} \subset D$ such that the transcendence degree of the field $K\left(D^{\prime}\right)\left(z_{0}, z_{1}, \cdots, z_{n}\right), y\left(x_{0}\right.$; $\left.z ; x), y^{\prime}\left(x_{0} ; z ; x\right), \cdots, y^{(n)}\left(x_{0} ; z ; x\right)\right)$ over $K\left(D^{\prime}\right)$ is $n$.

Proof. The equivalence of the conditions follows from tr. d. $\left[K\left(D^{\prime}\right)\left(z_{0}\right.\right.$, $\left.\left.z_{1}, \cdots, z_{n}\right): K\left(D^{\prime}\right)\right]=n$, tr. d. $\left[K\left(D^{\prime}\right)\left(y\left(x_{0}, z ; x\right), y^{\prime}\left(x_{0} ; z ; x\right), \cdots, y^{(n)}\left(x_{0} ; z ; x\right)\right.\right.$ : $\left.K\left(D^{\prime}\right)\right]=n$.

We introduce the notion of the algebraic dependence on the initial conditions. 
Definition (19). If the equivalent conditions of Lemma (18) are satisfied, we say that the general solution of the differential equation depends algebraically on the initial conditions.

We have a similar characterization as Proposition (16).

LEMma (19.1). The following conditions are equivalent.

(1) The general solution of the differential equation (6) depends algebraically on the initial conditions.

(2) There exist a general point $x_{0}$ and a subdomain $x_{0} \in D^{\prime} \subset D$ such that $\left(z_{0}, z_{1}, \cdots, z_{n}\right) \rightarrow\left(y\left(x_{0} ; z ; x\right), y^{\prime}\left(x_{0} ; z ; x\right), \cdots, y^{(n)}\left(x_{0} ; z ; x\right)\right)$ defines $K\left(D^{\prime}\right)$ algebraic correspondence between $X_{0} \otimes_{\mathrm{c}} K\left(D^{\prime}\right)$ and $\mathscr{X} \otimes_{R} K\left(D^{\prime}\right)$.

If the ring $R$ is of finite type over $\mathbb{C}$, then there conditions are equivalent to the following condition (3).

(3) There exist a general point $x_{0}$ and a subdomain $x_{0} \in D^{\prime} \subset D$, a $\mathbb{C}$-algebra $R^{\prime}$ consisting of holomorphic functions on $D^{\prime}$ containing $R$ and of finite type over $\mathbb{C}$ and an algebraic variety $\mathscr{Z} \subset X_{0} \times \mathscr{X} \otimes_{R} R^{\prime}=\left(X_{0} \times\right.$ Spec $\left.R^{\prime}\right) \times_{\text {Spec } R^{\prime}}\left(\mathscr{X} \times_{\text {Spec } R}\right.$ Spec $\left.R^{\prime}\right)$ such that (i) $\mathscr{Z}$ defines an $R^{\prime}$-algebraic correspondence between the algebraic varieties $X_{0} \otimes_{\mathrm{c}} \operatorname{Spec} R^{\prime}$ and $\mathscr{X} \times_{\operatorname{spec} R}$ Spec $R^{\prime}$ or equivalently at each point $u \in \operatorname{Spec} R^{\prime}$, the fibre $\mathscr{Z}_{u}$ defines an algebraic correspondence between $X_{0}$ and $\mathscr{X}_{u}$ and such that (ii) $\left(z_{0}, z_{1}, \cdots\right.$, $\left.z_{n}, y\left(x_{0} ; z ; x\right), y^{\prime}\left(x_{0} ; z ; x\right), \cdots, y^{(n)}\left(x_{0} ; z ; x\right)\right) \in X_{0} \times X_{i(x)}$ is in $\mathscr{Z}$ for $x \in D^{\prime}$ $\left(i: D^{\prime} \rightarrow\right.$ Spec $R^{\prime}$ being the natural morphism).

The proof is similar to that of Proposition (16) and is omitted.

As in the case of the rational dependence on the initial conditions, we can generalize Definition (19) to an abstract setting.

Definition (20). Let $L \supset K$ be an extension of ordinary differential field. We assume that the field extension $L \supset K$ is regular and of finite type. We say that the extension $L \supset K$ depends algebraically on the initial coditions if there exists a differential field extension $M \supset K$ such that the quotient field $N$ of $L \otimes_{K} M$ which is a differential field extension of $M$, is algebraic over a field generated over $M$ by the field $C_{N}$ of the constants.

Remark (20.1). It follows from the definition that for any differential field extension $J \supset K$, an extension $L \supset K$ depends algebraically on the initial conditions if and only if the base change $Q\left(L \otimes_{K} J\right) \supset J$ depends algebraically on the initial conditions (cf. Remark (11.1)). 
As in the case of the rational dependence, we can show the equivalence of Definitions (19) and (20) in the analytic setting.

\section{§ 2. Theorems on the reduction of a differential equation}

As there is an ambiguity in the statement of Theorem (5.3) of [U1] which says that we can solve an algebraic differential equation by a finite iteration of permissible operations if the general solution depends rationally on the initial conditions, we should clarify the assertion. The ambiguity comes from the word general. Let us clear away the defect. Let $K$ be a differential field and $F\left(Y_{0}, Y_{1}, \cdots, Y_{n}\right) \in K\{Y\}$ be an absolutely irreducible polynomial. The polynomial $F$ defines the prime differential ideal $\mathfrak{B}$ of the general component ([K], Chap, IV, $\S 6$, Theorem 3 ).

Definition (21). A generic solution of a differential equation $F=0$ over $K$ is a $K$-isomorphism $\varphi$ of the quotient differential field of the ring $K\{Y\} / \Re$ to an extension $L \supset K$ of the differential field $K$.

Since the isomorphism $\varphi$ is uniquely determined by the images $\varphi\left(Y_{0}\right), \varphi\left(Y_{1}\right), \cdots, \varphi\left(Y_{n}\right) \in L$ or even by $\varphi\left(Y_{0}\right)$, we call by abuse of language, $\left(\varphi\left(Y_{0}\right), \varphi\left(Y_{1}\right), \cdots, \varphi\left(Y_{n}\right)\right)$ or $\varphi\left(Y_{0}\right)$ a generic solution of $F=0$ over $K$.

ExAmple (21.1). Let $D$ be a domain and $K$ a differential field of meromorphic functions on $D$. Let $F\left(x ; Y_{0}, Y_{1}, \cdots, Y_{n}\right)=F\left(Y_{0}, Y_{1}, \cdots, Y_{n}\right)$ $\in K\{Y\}$ be an absolutely irreducible polynomial. By replacing $D$ by a smaller subdomain, we may assume that the coefficients of $F$ are regular on $D$. Let $x_{0} \in D$ be a general point so that (1) $X_{x_{0}}$ is irreducible and the function $\partial F / \partial Y_{n}\left(x_{0} ; Y_{0}, Y_{1}, \cdots, Y_{n}\right)$ on $X_{x_{0}}$ is not constantly equal to 0 . For any point of $X_{x_{0}}^{\prime}=\left\{z \in X_{x_{0}} \mid \partial F / \partial Y_{n}\left(x ; z_{0} ; z_{1}, \cdots, z_{n}\right) \neq 0\right\}$, there exists an open neighbourhood $V$ in $X_{x_{0}}$ for the usual topology and a neighbourhood $D^{\prime}$ of $x_{0}$ such that $\partial F / \partial Y_{n}\left(x ; z_{0}, z_{1}, \cdots, z_{n}\right) \neq 0$ if $\left(x ; z_{0}, z_{1}, \cdots, z\right)$ $\in D^{\prime} \times V$ and such that we can speak of the holomorphic solution $y\left(x_{0} ; z ; x\right)$ for $(z, x) \in U \times V$ with $y\left(x_{0} ; z ; x_{0}\right)=z_{0}, y^{(1)}\left(x_{0} ; z ; x_{0}\right)=z_{1}, \cdots$, $y^{(n)}\left(x_{0} ; z ; x_{0}\right)=z_{n}$. Taking $z$ as variable point in $U,\left(y\left(x_{0} ; z ; x\right), y^{(1)}\left(x_{0} ; z ; x\right)\right.$, $\left.\cdots, y^{(n)}\left(x_{0} ; z ; x\right)\right)$, which is customarily called the generic solution of $F=0$ in the analysis, is a generic solution of $F=0$ over $K(D)$ and hence over $K$. We may replace $D$ by any subdomain so far as it is a neighbourhood of the point $x_{0}$.

The objective of Painlevé $[\mathrm{P}]$ is the discovery of special functions defined by algebraic differential equations of order 2 . To examine whether 
he obtained essentially new functions, he had to treat the problem of reduction. For this purpose we should generate functions from the known functions. It is natural to start from the field $\mathbb{C}$ of the constant functions (cf. [U3]). The function should be meromorphic over a domain $D$ of the complex plane and parameters should take numerical values if there are any. The classical authors particularly Painlevé and J. Liouville are conscious that this idea is analogous to the number theory. In the number theory, we start from the field $\mathbb{Q}$ of the rational numbers and study algebraic and transcendental numbers in $\mathbb{C}$. Namely we have a correspondence:

Number theory

Analysis

$\mathbb{Q} \longleftrightarrow$ The field $\mathbb{C}$ of the constant functions on $D$

$\cap \cap \cap$

$\mathbb{C} \longleftrightarrow$ The field $K(D)$ of the meromorphic functions on $D$

From this point of view, the general solution $y\left(x_{0} ; z ; x\right)$ which contains a variable $z$ is incovenient. The initial conditions should not be a variable point but a $\mathbb{C}$-valued point of $X_{x_{0}}$.

Definition (22). Let $R$ be a differential ring of holomorphic functions on a domain $D \subset \mathbb{C}$ and $F\left(x ; Y_{0}, Y_{1}, \cdots, Y_{n}\right) \in R\{Y\}$ be an absolutely irreducible polynomial. Let $W \subset X_{x_{0}}$ be a non-empty dense open set for the usual topology of the algebraic variety $U=\left\{z \in X_{x_{0}} \mid \partial F / \partial Y_{n}\left(x_{0} ; z\right) \neq 0\right\}$ $\subset X_{x_{0}}$. By a solution of $F=0$ with general initial conditions at $x_{0}$, we mean any solution $y(x)$ whose initial conditions $\left(y\left(x_{0}\right), y^{\prime}\left(x_{0}\right), \cdots, y^{(n)}\left(x_{0}\right)\right)$ is in $W$.

TheOREm (23) ([N3]). Let $L \supset K$ be a regular extension of a differential field $K$. We assume that the field $L$ is finitely generated over $K$ as an abstract field and the field of the constants of $L$ coincides with that of $K$. If the field $C_{K}$ of the constants of $K$ is algebraically closed, then the following 2 conditions are equivalent.

(1) The extension $L \supset K$ depends rationally on the initial conditions.

(2) The extension $L \supset K$ is a subfield of a strongly normal extension of $K$ (for the definition see below).

If moreover the field $K$ is algebraically closed, then the above conditions (1) and (2) are equivalent to the following conditions.

(3) The extension $L \supset K$ is a subfield of a G-primitive extension of 
$K$ for an algebraic group $G$ defined over the field $C_{K}$ of the constants of $K$ (cf. [K2], [U1], [U3] and Definition (4), Proposition (5)).

Before we start the proof of the theorem, let us recall the definition of a strongly normal extension. We restrict ourselves to regular extensions.

Definition (23.0). A regular differential field extension $M \supset N$ is strongly normal if the following conditions are satisfied:

(i) The field extension $M \supset N$ is finitely generated;

(ii) The field of constants $C_{M}$ of $M$ coincides with $C_{N}$;

(iii) A differential field $Q\left(M \otimes_{N} M\right)$ is generated over $i_{2}(M)$ (which we sometimes denote by $M)$ by constants, where $i_{2}: M \rightarrow M \otimes_{N} M \subset Q\left(M \otimes_{N} M\right)$ is a morphism of differential fields defined by $i_{2}(m)=1 \otimes m$ for $m \in M$.

The equivalence of the conditions (2) and (3) of the theorem is a question of Galois cohomology and well known (cf. [K2], Chap. VI, in particular Corollary 2 (a), (b) on p. 425).

The theorem is essentially due to Nishioka. We give a proof of the theorem as we need Theorem (29) below which is proved by the same spirit as the above theorem. We considerably simplify the argument of Nishioka. In what follows, we assume that the extension $L \supset K$ is simple $(L=K\langle y\rangle)$ for the following two reasons. First, the extension is generated over $K$ by a finite number of elements $y_{1}, y_{2}, \cdots, y_{m} \in L$ as an abstract field. But in the proof, we have to consider not only the $y_{i}$ 's but also their derivatives and their higher derivatives. This fact makes the notation complicated yet the proof is essentially the same as the special case $L=K\langle y\rangle$. Secondly if $K \supset \mathbb{Q}(x)$ with $\delta(x)=1$, then the differential field $L$ is generated over $K$ by a single element by [K2], Chap. II, §8, Proposition 9.

Proof of Theorem (23). Let us show that the condition (2) implies the condition (1). If the condition (2) is satisfied, then there exists a differential field extension $M \supset K$ containing $L$ such that $Q\left(M \otimes_{K} M\right)$ is generated over $i_{2}(M)$ by constants. $Q\left(L \otimes_{N} M\right)$ is a differential subfield of $Q\left(M \otimes_{K} M\right)$. It follows from Proposition (12) that $Q\left(L \otimes_{K} M\right)$ is generated over $M$ by constants and the condition (1) is satisfied. It remains to show that the condition (1) implies the condition (2). It follows from the definition that there exists a differential field $M$ such that $Q\left(L \otimes_{K} M\right)$ 
is generated over $M$ by constants: there exist constants $c_{1}, c_{2}, \cdots, c_{r}$ such that $Q\left(L \otimes_{K} M\right)=M\left(c_{1}, c_{2}, \cdots, c_{r}\right)$. If $K\langle y\rangle=K\left(y, y^{\prime}, y^{(2)}, \cdots, y^{(s)}\right)$, then

$$
M\left(c_{1}, c_{2}, \cdots, c_{r}\right)=M\left(y, y^{\prime}, y^{(2)}, \cdots, y^{(s)}\right) .
$$

The geometric meaning of (23.1) is as follows. Let $Y=\operatorname{Spec} C_{M}\left[c_{1}, c_{2}, \cdots\right.$, $\left.c_{r}\right]$. We may assume that $C_{M\left(c_{1}, c_{2} \cdots c_{r}\right)}=C_{M}\left(c_{1}, c_{2}, \cdots, c_{r}\right)$ so that $M\left(c_{1}, c_{2}\right.$, $\left.\cdots, c_{r}\right)=Q\left(M \otimes_{C_{M}} C_{M}\left(c_{1}, c_{2}, \cdots, c_{r}\right)\right)$ by [K2], Chap. II, 1, Corollary 1 and hence $Y \otimes_{C_{M}} M$ is $M$-birationally equivalent to $\operatorname{Spec} M\left[y, y^{\prime}, y^{(2)}, \cdots, y^{(s)}\right]$. It follows from (23.1) that we can find polynomials $A_{i}\left(U_{1}, U_{2}, \cdots, U_{r}\right)$, $B\left(U_{1}, U_{2}, \cdots, U_{r}\right) \in M\left[U_{1}, U_{2}, \cdots, U_{r}\right]$ and $C_{j}\left(Y^{(0)}, Y^{(1)}, \cdots, Y^{(s)}\right), D\left(Y^{(0)}, Y^{(1)}\right.$, $\left.\cdots, Y^{(s)}\right) \in M\left[Y^{(0)}, Y^{(1)}, \cdots, Y^{(s)}\right]$ for $1 \leq i \leq s, 1 \leq j \leq r$ such that we have

$$
\begin{array}{ll}
y^{(i)}=A_{i}(c) / B(c) & \text { with } B(c) \neq 0 \\
c_{j}=C_{j}(\tilde{y}) / D(\tilde{y}) & \text { with } D(\tilde{y}) \neq 0
\end{array}
$$

for $1 \leq i \leq s, 1 \leq j \leq r$, where we denote $\left(c_{1}, c_{2}, \cdots, c_{r}\right)$ (resp. $\left(y, y^{\prime}, \cdots\right.$, $\left.\left.y^{(s)}\right)\right)$ by $c$ (resp. $\tilde{y}$ ). (23.2) defines an $M$-birational correspondence $\Phi$ : $Y \otimes_{C_{M}} M \cdots \operatorname{Spec} M\left[y, y^{\prime}, \cdots, y^{(s)}\right]$. Conversely let us notice that

(23.3) $Q\left(L \otimes_{K} M\right)$ is generated over $M$ by constants if and only if there exist polynomials $A_{i}, B, C_{j}, D$ and the constants $c_{j}$ 's satisfying the condition (23.2).

The proof is done in several steps.

(23.4) We may assume $C_{M}=C_{K}$.

Let $M_{0}$ be a differential algebra generated over $K$ by the coefficients of $C, D$ and of the $A_{i}$ 's and $C_{j}$ 's. In view of (23.3) we have to look for a $K$-morphism of differential algebras $f: M_{0} \rightarrow \bar{M}$ satisfying the following conditions :

(23.5) $\bar{M}$ is differential field extension of $K$;

(23.6) $C_{\bar{M}}=C_{K}$;

(23.7) $D^{f}(\tilde{y})\left(=D^{f}(y \otimes 1)\right)$ is not equal to 0 , where $D^{f}$ denotes the polynomial obtained from $D$ by applying to each coefficients a morphism $\mathrm{Id} \otimes f: L \otimes_{K} M_{0} \rightarrow L \otimes_{K} \bar{M}$

(23.8) Using the similar notation as in (23.7) and letting $\bar{c}_{j}=\left(C_{j} / D\right)^{f}(y)$ for $1 \leq j \leq r$, which are constants in $Q\left(L \otimes_{K} \bar{M}\right)$, we have $B_{i}^{f}\left(\bar{c}_{1}, \bar{c}_{2}, \cdots, \bar{c}_{r}\right)$ $\neq 0$.

Notice that if the conditions (23.6) and (23.7) are satisfied, we have necessarily $(\operatorname{Id} \otimes f)\left(y^{(i)}\right)=\left(A_{i} / B\right)^{f}(\bar{c})$ and $(\operatorname{Id} \otimes f)\left(c_{j}\right)=\left(C_{j} / D\right)^{f}(y \otimes 1)$.

We can find $f: M_{0} \rightarrow \bar{M}$ satisfying the conditions (23.5), (23.6), (23.7) 
and (23.8) by the following result of Ritt-Kolchin (Theorem 1 in [K1]).

THEOREM (24). Let $K$ be an (crdinary) differential field with an algebraically clesed field of constants $C_{K}$. Let $M_{0}$ be a differential K-algebra and $0 \neq u$ be element of $M_{0}$. We assume that the differential K-algebra $M_{0}$ is of finite type over $K$ (the differential ring $M_{0}$ is generated over $K$ by a finite number of elements, their derivatives and higher derivatives). Then there exists a K-morphism $f: M_{0} \rightarrow \bar{M}$ of the differential $K$-algebra $M_{0}$ to a differential over-field $\bar{M}$ of $K$ such that $f(u) \neq 0$ and $C_{K}=C_{\bar{M}}$.

(25.1) We may assume that $C_{M}=C_{K}$ and that there exist differential $K$-morphisms $f_{k}: K\{y\} \rightarrow M$ for $1 \leq k \leq l$ and polynomials $A_{i}, B \in M[U]$, $C_{j}, D \in M[Y](1 \leq i \leq s, 1 \leq j \leq r)$ satisfying the following conditions:

(25.1.0) The condition (23.2) (and hence the condition (23.1) also) holds;

(25.1.1) $D\left(\tilde{z}_{k}\right) \neq 0$ where $z_{k}=f_{k}(y)$ and $\tilde{z}_{k}=\left(z_{k}, z_{k}^{\prime}, \cdots, z_{k}^{(s)}\right)$ for $1 \leq k$ $\leq l$

(25.1.2) If we put $c_{j}^{(k)}=C_{j} / D\left(\tilde{z}_{k}\right)$, then $B\left(c_{1}^{(k)}, c_{2}^{(k)}, \cdots, c_{r}^{(k)}\right) \neq 0$ for $1 \leq k \leq l$;

(25.1.3) We have $z_{k}^{(j)}=A_{i} / B\left(c^{(k)}\right)$ for $0 \leq i \leq s$ and $c_{j}^{(k)}=C_{j} / D\left(\tilde{z}_{k}\right)$ for $1 \leq j \leq r$

(25.1.4) If we put $E=K\left\langle z_{k}\right\rangle_{1 \leq k \leq l} \subset M$, then $y \otimes 1 \in Q\left(K\langle y\rangle \otimes_{K} M\right)$ is in $C_{Q(K\langle y\rangle \otimes M)} \cdot E$, where $E \subset M$ is identified with a subfield of $K\langle y\rangle \otimes_{K} M$ $\subset Q\left(K\langle y\rangle \otimes_{K} M\right)$ by the canonical inclusion.

The condition (25.1.4) implies $E\langle y\rangle=C_{E\langle y\rangle} E$ by Proposition 12, here strictly speaking $y$ should be denoted by $y \otimes 1$ which would contribute to a notational complication.

Let us now denote $C_{K}=C_{M}$ by $C$ and $C\left[c_{1}, c_{2}, \cdots, c_{r}\right]$ by $C[c]$. Let $Y_{(m)}$ be the set of the $C$-rational points of $Y=\operatorname{Spec} C[c]$. In the language of E.G.A. Chap. I, $Y_{(m)}$ is the maximal spectra of $C[c] . \quad Y_{(m)}$ is the intuitive algebraic variety $Y$ (we notice that $C$ is algebraically closed).

Let $\mathscr{M}$ be a $C$-algebra and $f(c) \in \mathscr{M} \otimes_{c} C[c] \simeq \mathscr{M}[c]$. For $\bar{c} \in Y_{(m)}$, we denote by $f(\bar{c})$ the image of $f(c)$ by the $\mathscr{M}$-morphism $\mathscr{M} \otimes_{C}[C[c] \rightarrow \mathscr{M}$ induced by the $C$-morphism $C[c] \rightarrow C$ taking values at the $C$-rational point $\bar{c}$.

Sublemma (25.2). Let $U$ be a non-empty open set of $Y_{(m)}$. If $f(\bar{c})=0$ for any $\bar{c} \in U$, then $f(c)=0$. 
Proof of sublemma. Let $f(c)=\sum_{i=1}^{l} a_{i} \otimes b_{i}(c)$ with $a_{i} \in \mathscr{M}, b_{i} \in C[c]$. If $f \neq 0$, then we may assume that the $a_{i}$ 's (resp. $b_{j}$ 's) are linearly independent over $C$. Then $0=f(\bar{c})=\sum_{i=1}^{l} a_{i} \otimes b_{i}(\bar{c})$. Since the $a_{i}$ 's are linearly independent over $C$ and since $b(\bar{c}) \in C$, we conclude $b_{i}(\bar{c})=0$ for any $\bar{c} \in U$ and hence $b_{i}=0$ which is a contradiction.

Corollary (25.3) to Sublemma. Let $0 \neq f \in \mathscr{M}[c]$. Then $\left\{\bar{c} \in Y_{(m)} \mid f(\bar{c})\right.$ $=0\}$ is a proper closed subset of $Y_{(m)}$ if it is not empty.

Proof of the Corollary. In fact if $f=\sum_{i=1}^{l} a_{i} \otimes b_{i}$ with the $a_{i}$ 's (resp. the $b_{i}$ 's) linearly independent over $C$, then the above argument shows $\left\{\bar{c} \in Y_{(m)} \mid f(\bar{c})=0\right\}=\left\{\bar{c} \in Y_{(m)} \mid b_{i}(\bar{c})=0\right.$ for $\left.1 \leq i \leq l\right\}$. Hence the subset is closed. It follows from the sublemma that this subset can not be the whole space $Y_{(m)}$.

Corollary (25.4) тo Sublemma. $A_{i}, B, C_{j}$ and $D$ being as in (23.2), there exist a non-empty Zariski open set $U$ of $Y_{(m)}$ satisfying the following conditions;

(1) If $\bar{c} \in U$, then the morphism $M[c] \rightarrow M$ induced by $\bar{c}$ can be extended to $M[c]_{B(c)} \rightarrow M\left(M[c]_{B[c]}\right.$ is the localization of $M[c]$ with respect to $B(c))$;

(2) If $\bar{c} \in U$, denoting $y(\bar{c}), y^{\prime}(\bar{c}), \cdots, y^{(s)}(\bar{c}) \in M$ be the images of $y(c)$, $y^{\prime}(c), \cdots, y^{(s)}(c) \in M[c]_{B[c]}$ by the above morphism, we have

$$
\begin{aligned}
& D\left(y(\bar{c}), y^{\prime}(\bar{c}), \cdots, y^{(s)}(\bar{c})\right) \neq 0, \\
& \bar{y}^{(i)}(\bar{c})=A_{i} / B\left(\bar{c}_{1}, \bar{c}_{2}, \cdots, \bar{c}_{r}\right) \quad \text { and } \\
& \bar{c}_{j}=C_{j}{ }_{j}^{\prime} D\left(y(\bar{c}), y^{\prime}(\bar{c}), \cdots, y^{(s)}(\bar{c})\right)
\end{aligned}
$$

for $0 \leq i \leq s, 0 \leq j \leq r$.

Proof of Corollary (25.4), The first condition is satisfied if $B(\bar{c}) \neq 0$ and therefore on a non-empty open set $V$ of $Y_{(m)}$ by Corollary (25.3). As for the second condition for sufficiently large $N$, we have $G(c)=$ $B(c)^{N} D\left(y, y^{\prime}, \cdots, y^{(s)}\right)$ is in $M[c]$. Therefore on a non-empty open set $W$ of $Y_{(m)}$, the function $G(c)$ never vanishes by Corollary (25.3). Now it is sufficient to take $U=V \cap W$.

Sublemma (25.5). Let $F \supset k$ be a field extension and $a$ be an element of $F$. Then the following conditions are equivalent.

(1) The element $a$ is in $k$.

(2) $\varphi_{1}(a)=\varphi_{2}(a)$ where $\varphi_{1}$ (resp. $\left.\varphi_{2}\right)$ is a morphism of $F$ into $F \otimes_{k} F$ 
defined by $\varphi_{1}(b)=b \otimes 1\left(\right.$ resp. $\left.\varphi_{2}(b)=1 \otimes b\right)$ for $b \in F$.

Proof. It is evident that the condition (1) implies (2). Conversely the condition (2) implies (1) since any $k$-module is free.

Let us come back to the proof of (25.1). Using the open set $U$ of Corollary (25.4), we put $\mathscr{E}=K\langle y(\bar{c})\rangle_{\bar{c} \in U}$. We show that $y$ is in $\mathscr{E}\left(c_{1}, c_{2}\right.$, $\left.\cdots, c_{r}\right)\left(\subset Q\left(L \otimes_{K} M\right)=M\left(c_{1}, c_{2}, \cdots, c_{r}\right)\right)$. In fact this implies that there exist a finite number of points $\bar{c}^{(1)}, \bar{c}^{(2)}, \cdots, \bar{c}^{(l)}$ of $U$ such that $y$ is in a differential field $K\left\langle y\left(\bar{c}^{(k)}\right)\right\rangle_{1 \leq k \leq l}\left(c_{1}, c_{2}, \cdots, c_{r}\right)$. Let $E=K\left\langle y\left(\bar{c}_{k}\right)\right\rangle_{1 \leq k \leq l}$. If we take $A_{i}, B, C_{j}$ and $D$ as in Corollary (25.4), then the conditions (25.1.1), (25.1.2), (25.1.3) and (25.1.4) are satisfied. Let $\varphi_{i}: M(c) \rightarrow M(c) \otimes_{\varepsilon(c)} M(c)$ $(i=1,2)$ be two morphisms defined by $\varphi_{1}(a)=a \otimes 1, \varphi_{2}(a)=1 \otimes a$ for $a \in M(c)$. In view of Sublemma (25.5), it is sufficient to show that $\varphi_{1}(y)$ $=\varphi_{2}(y)$ or $y \otimes 1-1 \otimes y$ is equal to zero in $M(c) \otimes_{{ }_{8(c)}} M(c) . \quad y \otimes 1-1 \otimes y$ in question is an image of $y \otimes 1-1 \otimes y$ in $\left(M[c] \otimes_{\varepsilon[c]} M[c]\right)_{B(c) \times B(c)}$ and hence it is sufficient to show $y \otimes 1-1 \otimes y=0$ in $\left(M[c] \otimes_{\delta(c)} M[c]\right)_{B(c) \otimes B(c)}$. Since $M[c]$ is faithfully flat over $\mathscr{E}[c], B(c) \otimes B(c)$ is not a zero divisor in $M[c] \otimes_{\delta[c]} M[c]$. Therefore it is sufficient to show that $B(c) \otimes B(c)(y \otimes 1$ $-1 \otimes y)=0$ in $M[c] \otimes_{\sigma[c]} M[c] \simeq\left(M \otimes_{\delta} M\right) \otimes_{c} C[c]$. By Sublemma (25.2) it is sufficient to show that $B(c) \otimes B(c)(y \otimes 1-1 \otimes y)(\bar{c})=0$ for any $\bar{c} \in U$. Since $B(\bar{c}) \otimes B(\bar{c})$ is not a zero divisor in $M[\bar{c}] \otimes_{\mathscr{\delta}[\bar{c}]} M[\bar{c}]$ (notice $\left.\mathscr{E}[\bar{c}]=\mathscr{E}\right)$, the latter condition is equivalent to $y(\bar{c}) \otimes 1-1 \otimes y(\bar{c})=0$ in $\left(M[\bar{c}] \otimes_{\bullet[\bar{c}]}\right.$ $M[\bar{c}])$. This is so since $y(\bar{c})$ is in $\mathscr{E}=K\langle y\rangle_{\bar{c} \in U}$.

(26.1) In addition to the requirements of (25.1), we may assume that the differential field $K\langle y\rangle$ is $K$-isomorphic to $K\left\langle z_{1}\right\rangle$.

In fact let us consider an extension $\tilde{L}=Q\left(L \otimes_{K} L\right)$ of $i_{2}(L)=L_{2}$ where $i_{2}: L \rightarrow L \otimes_{k} L \subset Q\left(L \otimes_{K} L\right)$ is defined by $i_{2}(a)=1 \otimes a$ for $a \in L$. The extension $\tilde{L} \supset L_{2}$ depends rationally on the initial conditions by Remark (11.1). Therefore we can find $\mathscr{M} \supset L_{2}$ and $L_{2}$-morphisms $\tilde{f}_{i}: L_{2}\{y\} \rightarrow \mathscr{M}$ $(1 \leq i \leq k)$ and polynomial with $z_{i}=\tilde{f}_{i}(y)$ satisfying (25.1.1), (25.1.2) and (25.1.3) over $L_{2}$. It is sufficient to take $\mathscr{M}$ for $M$, the polynomials for $L_{2}\langle y\rangle / L_{2}$ and $K$-morphisms $f_{i}: K\{y\} \rightarrow \mathscr{M}$ induced by $\tilde{f}_{i}$.

Here is the conclusion from (25.1) and (26.1). By (25.1.4) we may assume $A_{i}, B \in E[c], C_{j}, D \in E[y]$ and now taking $E$ for $M$, we can find a differential field extension $M$ of $K$ and $z, z_{1}, z_{2}, \cdots, z_{l} \in M$ satisfying the following conditions (see Remark (28) below).

(27.0) $\quad M=K\left\langle z, z_{1}, z_{2}, \cdots, z_{l}\right\rangle$. 
(27.1) The field of the constants of $M$ coincides with that of $K$.

(27.2) A differential subfield $K\langle z\rangle$ of $M$ is $K$-isomorphic to $K\langle y\rangle$.

(27.3) $Q\left(L \otimes_{K} M\right)$ is generated over $M$ by the constants; $Q\left(L \otimes_{k} M\right)$ $=M\left(c_{1}, c_{2}, \cdots, c_{r}\right), c_{1}, c_{2}, \cdots, c_{r}$ being constants.

(27.4) $y^{(i)}(c)=A_{i}(c) / B(c)$, with $A_{i}(c), B(c) \in M\left[c_{1}, c_{2}, \cdots, c_{r}\right]$ for $0 \leq i$ $\leq s$ and $c_{j}=C_{j}\left(y, y^{(1)}, \cdots, y^{(s)}\right) / D\left(y, y^{(1)}, \cdots, y^{(s)}\right)$ with $C_{j}\left(y, y^{(1)}, \cdots, y^{(s)}\right)$, $D\left(y, y^{(1)}, \cdots, y^{(s)}\right) \in M\left[y, y^{(1)}, \cdots, y^{(s)}\right]$ for $1 \leq j \leq r$.

(27.5.1) There exist $C$-morphisms $C\left[c_{1}, c_{2}, \cdots, c_{r}\right] \rightarrow C,(c) \rightarrow\left(\bar{c}^{(k)}\right)$ for $1 \leq k \leq l$. Since $M\left[c_{1}, c_{2}, \cdots, c_{r}\right] \simeq M \otimes_{C} C\left[c_{1}, c_{2}, \cdots, c_{r}\right]$ by [K2], Chap. II, $\S 1$, Corollary 1 , these morphisms define $M$-morphisms $M\left[c_{1}, c_{2}, \cdots, c_{r}\right] \rightarrow M$ such that $B\left(\bar{c}^{(k)}\right) \neq 0$ and such that $y^{(j)}\left(\bar{c}^{(k)}\right)=A_{j}\left(\bar{c}^{(k)}\right) / B\left(\bar{c}^{(k)}\right)=z_{k}^{(j)}$ for $1 \leq k \leq l, 0 \leq j \leq s$,

(27.5.2) Moreover $D\left(y\left(\bar{c}^{(k)}\right), y^{\prime}\left(\bar{c}^{(k)}\right), \cdots, y^{(s)}\left(\bar{c}^{(k)}\right)\right)=D\left(z_{k}, z_{k}^{\prime}, \cdots, z_{k}^{(s)}\right) \neq 0$ for $1 \leq k \leq l$ and $\bar{c}_{j}^{(k)}=C_{i}\left(z_{k}, z_{k}^{\prime}, \cdots, z_{k}^{(s)}\right) / D\left(z_{k}, z_{k}^{\prime}, \cdots, z_{k}^{(s)}\right)$ for $1 \leq j \leq s$ and for $1 \leq k \leq l$.

By (27.0), (27.1), $\cdots,(27.5)$ it is sufficient to show that $M=K\left(z, z_{1}\right.$, $\cdots, z_{l}$ ) is a strongly normal extension of $K$. Let us show that an extension $Q\left(M \otimes_{K} M\right)$ of $\varphi_{2}(M)$ is generated by constants, where $\varphi_{2}: M \rightarrow M \otimes_{K} M$ $\subset Q\left(M \otimes_{K} M\right)$ denotes a morphism defined by $a \rightarrow 1 \otimes a$ for $a \in M$. If we write $y=z \otimes 1$, then $K\langle y\rangle \cdot \varphi_{2}(M)$ is isomorphic to $Q\left(L \otimes_{K} M\right)$. Therefore identifying $M$ with $\varphi_{2}(M)$ by $\varphi_{2}$, we can find constants $c_{1}, c_{2}, \cdots, c_{r} \in$ $C_{K\langle y\rangle \varphi_{2}(M)}=C_{Q\left(L \otimes_{K} M\right)}, A_{i}, B, \varphi_{2}(M)[c]$ and $C_{j} . D \in \varphi_{2}(M)\left[y, y^{\prime}, \cdots, y^{(s)}\right]$ satisfying the following conditions

(27.6) $B(c) \neq 0$ and $y^{(i)}(c)=A_{i} / B(c)$ for $1 \leq i \leq s$;

(27.7) $D\left(y, y^{\prime}, \cdots, y^{(s)}\right) \neq 0$ and $c_{j}=C_{j} l D\left(y, y^{\prime}, \cdots, y^{(s)}\right)$ for $1 \leq i \leq r$. In particular $y=z \otimes 1$ is in $C_{Q\left(M \otimes_{K} M\right)} \cdot \varphi_{2}(M)$. Since $D\left(1 \otimes z_{k}, 1 \otimes z_{k}^{\prime}, \cdots, 1\right.$ $\left.\otimes z_{k}^{(s)}\right) \neq 0$ by (27.4) and since the $K$-morphism $K\left\{z_{k} \otimes 1\right\} \rightarrow K\left\{1 \otimes z_{k}\right\} \subset$ $\varphi_{2}(M)\left(z_{k} \otimes 1 \rightarrow 1 \otimes z_{k}\right)$ induces an $M$-morphism $K\left\{z_{k} \otimes 1\right\} \varphi_{2}(M) \simeq K\left\{z_{k} \otimes 1\right\}$ $\otimes_{K} \varphi_{2}(M) \rightarrow \varphi_{2}(M), D\left(z_{k} \otimes 1, z_{k}^{\prime} \otimes 1, \cdots, z_{k}^{(s)} \otimes 1\right) \neq 0$ and hence we can define $c_{j}^{(k)}=C_{j} / D\left(z_{k} \otimes 1, z_{k}^{\prime} \otimes 1, \cdots, z_{k}^{(s)} \otimes 1\right)$. It follows from (27.4) and (27.5.1) that we have a $\varphi_{2}(M)$-morphism $K\{y\} \cdot \varphi_{2}(M) \rightarrow \varphi_{2}(M), y=z \otimes 1 \rightarrow z_{k}$ and hence a $K$-morphism $K\{y\} \rightarrow \varphi_{2}(M)=M$. Therefore we have a $K$ morphism $K\{y\} \rightarrow K\left\{z_{k} \otimes 1\right\}$ by (27.2) which induces a $\varphi_{2}(M)$-morphism $K\{y\} \varphi_{2}(M) \simeq K\{y\} \otimes_{K} \varphi_{2}(M) \rightarrow K\left\{z_{k} \otimes 1\right\} \varphi_{2}(M)=\varphi_{2}(M)\left\{z_{k} \otimes 1\right\}$. This defines a $\varphi_{2}(M)$-morphism $\varphi_{2}(M)\left\{z \otimes 1, C_{0}(z \otimes 1) / D(z \otimes 1)\right\} \rightarrow \varphi_{2}(M)\left\{z_{k} \otimes 1, C_{0}\left(z_{k} \otimes 1\right)\right.$ $\left.\mid D\left(z_{k} \otimes 1\right)\right\}$ and hence the $c_{j}^{(k)}$ 's are constants in $Q\left(M \otimes_{K} M\right)$. Since $B\left(1 \otimes \bar{c}_{1}^{(k)}, 1 \otimes \bar{c}_{2}^{(k)}, \cdots, 1 \otimes \bar{c}_{r}^{(k)}\right) \neq 0$ by $(27.5 .2)$ and since we have $\varphi_{2}(M)$ - 
morphisms $\varphi_{2}(M)\left\{z_{k} \otimes 1\right\} \rightarrow \varphi_{2}(M)\left(z_{k} \otimes 1 \rightarrow 1 \otimes z_{k}\right)$. Therefore we have a $\varphi_{2}(M)$-morphism

$$
\begin{aligned}
& \varphi_{2}(M)\left\{y, \bar{c}_{1}, \bar{c}_{2}, \cdots, \bar{c}_{r}, A_{0} / B(\bar{c}), A_{1} / B(\bar{c}), \cdots, A_{s} / B(\bar{c})\right\} \rightarrow \\
& \varphi_{2}(M)\left\{z_{k} \otimes 1, \bar{c}_{1}^{(k)}, \bar{c}_{2}^{(k)}, \cdots, \bar{c}_{r}^{(k)}, A_{0} / B\left(\bar{c}^{(k)}\right), A_{1} / B\left(\bar{c}^{(k)}\right), \cdots, A_{s} / B\left(\bar{c}^{(k)}\right)\right\}
\end{aligned}
$$

for $1 \leq k \leq l$, we get $z_{k} \otimes 1=A_{0} / B\left(\bar{c}_{1}^{(k)}, \bar{c}_{2}^{(k)}, \cdots, \bar{c}_{r}^{(k)}\right)$ and $z_{k} \otimes 1$ is in $\varphi_{2}(M)\left(\bar{c}_{1}^{(k)}, \bar{c}_{2}^{(k)}, \cdots, \bar{c}_{r}^{(k)}\right) \subset C_{Q\left(L \otimes_{K} L\right)} \varphi_{2}(M)$.

Remark (28). In the last reduction the following fact is used.

Let $A(c), B(c) \in M[c]$ and $\bar{c} \in Y_{(m)}$ such that $B(\bar{c}) \neq 0$. If $A / B(c) \in M(c)$ lies in $E(c)$, then there exist $\mathscr{A}(c), \mathscr{B}(c) \in E[c]$ such that $A / B(c)=\mathscr{A} / \mathscr{B}(c)$ with $\mathscr{B}(\bar{c}) \neq 0$.

Let us see the proof of this fact. Let $m=\{f(c) \in M[c] \mid f(\bar{c})=0\}$ and $\mathfrak{m}=\{f(c) \in E[c] \mid f(\bar{c})=0\}$. Since $M[c] \simeq E[c] \otimes_{E} M$ and since $E$ is a field, $M[c]$ is flat over $E[c]$. Hence the local ring $M[c]_{m}$ is flat over $E[c]_{\mathrm{m}}$. Since $A / B \in M[c]_{m}$ is in $E(c)$, therefore there exist $\tilde{A}, \tilde{B} \in E[c]$ such that $A / B=\tilde{A} / \tilde{B}: \tilde{A}=(A / B) \tilde{B}$. Namely we have $\tilde{A}=(A / B) \tilde{B} \in E[c]_{\mathfrak{m}} \cap \tilde{B}\left(M[c]_{m}\right)$ which is equal to $\tilde{B}\left(E[c]_{\mathfrak{m}}\right)$ since $M[c]_{m}$ is flat over $E[c]_{\mathfrak{m}}$ (cf. [B], Chap. I, $\S 2,6$, Corollaire). Consequently we have $A / B=\tilde{A} / \tilde{B}=\tilde{C} \in E[c]_{\mathrm{m}}$.

The argument of (25.1) and (26.1) gives us the following result.

TheOREM (29). Let $\mathbb{C} \subset K$ be a field of known meromorphic functions on a domain $D$. If the general solution of the differential equation (6) depends rationally on the initial conditions, then there exist solutions $f_{1}, f_{2}$, $\cdots, f_{r}$ of (6) meromorphic on a subdomain of $D$ such that $y\left(x_{0} ; z ; x\right) \in$ $K\left\langle f_{1}, f_{2}, \cdots, f_{r}\right\rangle\left(z_{0}, z_{1}, \cdots, z_{n}\right)$ (here we denote by $y\left(x_{0} ; z ; x\right)$ the general solution of (6) taking the initial conditions $z=\left(z_{0}, z_{1}, \cdots, z_{n}\right)$ at $x_{0}$ and hence $z_{0}, z_{1}, \cdots, z_{n}$ are constants) and such that each of the solutions $f_{i}$ 's is obtained from $K$ by a finite iteration of the permissible operations $(\mathrm{O})$,

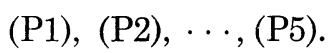

Since we can prove the theorem similarly as Theorem (23), we content ourselves with an outline of a proof. First let us notice that the extension $K\left\langle y\left(x_{0} ; z ; x\right)\right\rangle \supset K$ may have a non-trivial constant field extension. It follows from the definition that we can characterize the rational dependence by the birational correspondence (23.2). Therefore if we take a particular solution $y\left(x_{0} ; \bar{z} ; x\right)$ with general initial conditions $\bar{z}_{1}, \bar{z}_{2}, \cdots, \bar{z}_{n}$ at $x_{0}$, then the extension $K\left\langle y\left(x_{0} ; \bar{z} ; x\right)\right\rangle \supset K$ depends rationally on the 
initial conditions by the argument in (23). Hence by Theorem (23) $K<$ $\left.y\left(x_{0} ; \bar{z} ; x\right)\right\rangle$ is contained in a strongly normal extension of $K$. Hence extending $K$ algebraically if necessary, we may assume $K\left\langle y\left(x_{0} ; \bar{z} ; X\right)\right\rangle$ is in a $G$-primitive extension of $K$. Therefore $y\left(x_{0} ; \bar{z} ; x\right)$ is obtained from $K$ by a finite iteration of the permissible operations (O), (P1), (P2), . , (P5) by Theorem (2) and Theorem (3). The argument of (25.1) shows that if we know a finite number of solutions $f_{1}, f_{2}, \cdots, f_{m}$ with general initial conditions, $K\left\langle f_{1}, f_{2}, \cdots, f_{m}\right\rangle\left\langle y\left(x_{0} ; z ; y\right)\right\rangle$ is generated over $K\left\langle f_{1}, f_{2}, \cdots, f_{m}\right\rangle$ by constants.

To make our assertions and arguments clear, let us prove the following result which originated also in the Stockholm lessons. In fact the following theorem is a Corollary of Theorem (29).

TheOREM (30). Let $R$ be a differential ring of holomorphic functions on a domain $D \subset \mathbb{C}$ and $F\left(x ; Y_{0}, Y_{1}, \cdots, Y_{n}\right) \in R\left[Y_{0}, Y_{1}, \cdots, Y_{n}\right]$ be an $a b$ solutely irreducible polynomial. If the general solution of an algebraic differential equation $F\left(x ; y, y^{\prime}, \cdots, y^{(n)}\right)=0$ depends rationally on the initial conditions, then any solution with general initial conditions is obtained from $R$ by a finite iteration of the permissible operations (O), (P1), . , (P5).

Theorems (29) and (30) clarify Corollary (4.6) in [U1] and the same clarification should be done for Theorem (3.21) in [U1]. More generally we can prove

TheOREM (31). Let $R$ be a differential ring of holomorphic functions on a domain $D \subset \mathbb{C}$ and $F\left(x ; Y_{0}, Y_{1}, \cdots, Y_{n}\right) \in R\{Y\}$ be an absolutely irreducible polynomial. If the general solution of an algebraic differential equation $F\left(x ; y, y^{\prime}, \cdots, y^{(n)}\right)=0$ depends algebraically on the initial conditions, then any solution with general initial conditions is obtained from $R$ by a finite iteration of the permissible operations $(\mathrm{O}),(\mathrm{P} 1), \cdots,(\mathrm{P} 5)$.

We reduce this theorem to Theorem (29). We can find an idea of the reduction in the Leçons de Stockholm. Nishioka [N2] formulated the idea in an algebraic context and made it transparent. We complete his result to make its application easy. In fact we prove the theorem in [N2] under a weaker assumption and moreover our proof is simpler. However we are inspired by [P] and [N2]. 
Definition (32.1) ([N2], [K2]). A multi-index is an infinite sequence $J=\left(j_{0}, j_{1}, j_{2}, \cdots\right)$ of non-negative integers $j_{n}(n \in N)$ such that the $j_{n}$ 's are equal to 0 except for a finite number. Let $I=\left(i_{n}\right)$ and $J=\left(j_{n}\right)$ be two multi-indices. We say that the index $I$ is lower than the index $J$ or the index $J$ is higher than the index $I$ if there exists an integer $m$ with $i_{m}<j_{m}$ and $i_{n}=j_{n}$ for $n>m$. Let $M$ be a differential field and $M\{Y\}$ be a differential polynomial ring. Let $A \in M\{Y\}$. Then $A$ can be written in the form $\sum_{J} a_{J} Y^{J}$, where we denote by $Y^{J}$ a monomial $\prod_{n=0}^{\infty} Y_{n}^{j_{n}}$ and $J$ runs through the set of the multi-indices and the $a_{J}$ 's are equal to 0 except for finite indices.

The rank of the non-zero differential polynomial $A(Y)=\sum_{J} a_{J} Y^{J}$ is the highest multi-index $J$ such that $a_{J} \neq 0$.

Let $N \supset M$ be an extension of the differential field $M$ and $y \in N$. If tr. d. $[M\langle y\rangle: M]$ is finite $(M\langle y\rangle$ denotes a differential subfield of $N$ generated by $y$ over $M$ ), then there exists a non-zero differential polynomial $A(Y)$ such that $A(y)=0$. Among such polynomials, there exists one $A(Y)=\sum_{J} a_{J} Y^{J}$ which is of minimal rank. This polynomial is unique up to multiplication by an element of $K$ and hence is uniquely determined under the additional assumption $A_{I}=1$ for the highest multiindex $I$.

Definition (32.2). We call this unique polynomial the minimal polynomial of $y$ over $M$. Let $C$ be the field of the constants of $M\langle y\rangle$. We call the minimal polynomial of $y$ over $M C$ the characteristic polynomial of $y$ over $M$ and denote it by $\operatorname{Ch}(y ; M)$, where $M C$ is a differential field generated by $M$ and $C$.

Lemma (33). Let $M\langle y\rangle \supset M^{\prime} \supset M$ be a differential intermediate field. Then the following conditions are equivalent.

(1) $M=M^{\prime}$.

(2) The minimal polynomial of $y$ over $M^{\prime}$ coincides with the minimal polynomial of $y$ over $M$.

Proof. We must show that the condition (2) implies (1). Let $F^{\prime}\left(Y_{0}\right.$, $\left.Y_{1}, \cdots, Y_{m}\right)$ with $\partial F^{\prime} / \partial Y_{m} \neq 0$ be the minimal polynomial of $y$ over $M^{\prime}$ and $F\left(Y_{0}, Y_{1}, \cdot \cdot, Y_{n}\right)$ with $\partial F / \partial Y_{n} \neq 0$ be the minimal polynomial of $y$ over $M$. If $F=F^{\prime}$, then since $m=$ tr. d. $[M\langle y\rangle ; M]=$ tr. d. $\left[M^{\prime}\langle y\rangle ; M^{\prime}\right]$ and $n=\operatorname{tr}$. d. $[M\langle y\rangle ; M]$, the assumption implies that $m=n$ and that $y, y^{\prime}$, $\cdots, y^{(n-1)}$ are transcendental over $M^{\prime}$ and $y^{(n)}$ is algebraic over $M(y$, 
$\left.y^{\prime}, \cdots, y^{(n-1)}\right) ; M\left(y, y^{\prime}, \cdots, y^{(n-1)}\right) \subset M^{\prime}\left(y, y^{\prime}, \cdots, y^{(n-1)}\right) \subset M\left(y, y^{\prime}, \cdots, y^{(n)}\right)$. Therefore $M^{\prime}\left(y, y^{\prime}, \cdots, y^{(n-1)}\right)$ is algebraic over $M\left(y, y^{\prime}, \cdots, y^{(n-1)}\right)$, since $y, y^{\prime}, \cdots, y^{(n-1)}$ is transcendental. As $F\left(y, y^{\prime}, \cdots, y^{(n-1)}, Y^{(n)}\right)$ and $F^{\prime}\left(y, y^{\prime}\right.$, $\left.\cdots, y^{(n-1)}, Y^{(n)}\right)$ are respectively the minimal polynomials of $y^{(n)}$ over $M\left(y, y^{\prime}, \cdots, y^{(n-1)}\right)$ and $M^{\prime}\left(y, y^{\prime}, \cdots, y^{(n-1)}\right)$, we conclude $M\left(y, y^{\prime}, \cdots, y^{(n-1)}\right)$ $=M^{\prime}\left(y, y^{\prime}, \cdots, y^{(n-1)}\right)$. Hence $M=M^{\prime}$.

Let $L \supset K$ be a differential field extension. We assume that the field extension $L \supset K$ is differentially generated by a simple element $y$ over $K ; L=K\langle y\rangle$ (cf. [K2], Chap. §8, Proposition 9). Let $M \supset K$ be an differential field extension. We have a diagram.

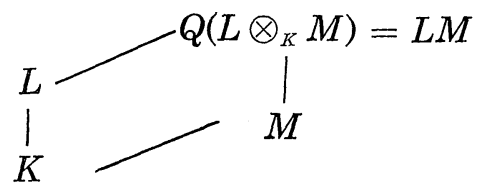

We identify $L$ and $M$ with subfields in $Q\left(L \otimes_{K} M\right)$. In this situation, we denote the characteristic polynomial $\mathrm{Ch}(y ; M)$ by $\mathrm{Ch}(y ; M / K)$.

LemMa (34). We have an inequality:

rank of $\mathrm{Ch}(y ; K) \geq \operatorname{rank}$ of $\mathrm{Ch}(y ; M / K)$.

Proof. The assertion follows from the inclusion $K C_{L}\{Y\} \subset M C_{L M}\{Y\}$ and the definition.

Corollary (35). There exists among differential field extension of $K$ an extension $M \supset K$ such that the rank of $\mathrm{Ch}(y ; M / K)$ is minimal.

As we see below, the field $M \supset K$ giving the minimal rank is not unique but the characteristic polynomial $\mathrm{Ch}(y ; M / K)$ is uniquely determined.

LemMa (36). Let $M_{1} \supset M_{2} \supset K$ be differential field extensions such that the rank of $\mathrm{Ch}\left(y ; M_{2} / K\right)$ is minimal. Then $\mathrm{Ch}\left(y ; M_{1} / K\right)=\mathrm{Ch}\left(y ; M_{2} / K\right)$. Here we identify $Q\left(L \otimes_{K} M_{2}\right)$ with a subfield of $Q\left(L \otimes_{K} M_{1}\right)$ and hence $Q\left(L \otimes_{K} M_{2}\right)\{Y\}$ with a subring of $Q\left(L \otimes_{K} M_{1}\right)\{Y\}$ by the inclusion $M_{2} \subset M_{1}$.

Proof. The characteristic polynomial $\mathrm{Ch}\left(y ; M_{2} / K\right)[Y] \in M_{2} C_{L M_{2}}\{Y\} \subset$ $M_{1} C_{L M_{1}}\{Y\}$ satisfies $\mathrm{Ch}\left(y ; M_{2} / K\right)(y)=0$. It follows from the definition of the minimal polynomial $\mathrm{Ch}\left(y ; M_{1} / K\right)$, rank of $\mathrm{Ch}\left(y ; M_{1} / K\right) \leq \operatorname{rank}$ of $\mathrm{Ch}\left(y ; M_{2} / K\right)$. By the minimality of the rank of $\mathrm{Ch}\left(y ; M_{2} / K\right)$, we have rank of $\mathrm{Ch}\left(y ; M_{2} / K\right) \leq$ rank of $\operatorname{Ch}\left(y ; M_{1} / K\right)$. Therefore the rank of 
$\operatorname{Ch}\left(y ; M_{1} / K\right)$ is equal to the rank of $\operatorname{Ch}\left(y ; M_{2} / K\right)$. Since the minimal polynomial is unique, we have $\operatorname{Ch}\left(y ; M_{1} / K\right)=\operatorname{Ch}\left(y ; M_{2} / K\right)$.

Corollary (37). The characteristic polynomial $\mathrm{Ch}(y ; M / K)$ of minimal rank is uniquely determined and independent of the field $M \supset K$.

Definition (38). We call the characteristic polynomial in the corollary the absolute characteristic polynomial of $y$ over $K$ and denote it by $\mathrm{CH}(y / K)$.

The absolute characteristic polynomial $\mathrm{CH}(y / K)$ is a polynomial with coefficients in $Q\left(L \otimes_{k} M\right)$ for suitable differential field extension $M \supset K$.

Proposition (39). The coefficients of the absolute characteristic polynomial $\mathrm{CH}(y / K)$ are in $L$.

Proof. First we assume that $K$ is algebraically closed. Let $M \supset K$ be a differential field extension such that $\operatorname{Ch}(y ; M / K)$ is the absolute characteristic polynomial $\mathrm{CH}(y / K)$. We have two isomorphisms $i_{1}$ and $i_{2}$ of $M$ into $M \otimes_{K} M \subset Q\left(M \otimes_{K} M\right) ; i_{1}(a)=a \otimes 1, i_{2}(a)=1 \otimes a$ for $a \in M$. Therefore we have two isomorphisms of $L \otimes_{K} M$ to $L \otimes_{K}\left(M \otimes_{K} M\right)=$ $\left(L \otimes_{K} M\right) \otimes_{L}\left(L \otimes_{K} M\right)$ which induces two isomorphisms $j_{1}$ and $j_{2}: Q\left(L \otimes_{K} M\right)$ $\rightarrow Q\left(L \otimes_{K} M\right) \otimes_{L} Q\left(L \otimes_{K} M\right) \subset Q\left(\left(L \otimes_{K} M\right) \otimes_{L}\left(L \otimes_{K} M\right)\right)=Q\left(L \otimes_{K}\left(M \otimes_{K} M\right)\right) ;$ $j_{1}(a)=a \otimes 1, j_{2}(a)=1 \otimes a$ for $a \in Q\left(L \otimes_{K} M\right)$. We have a commutative diagram:

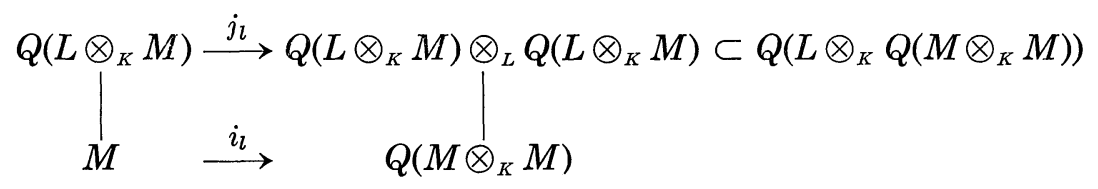

for $l=1$, 2. The polynomials $\mathrm{CH}^{j l}(y / K)$ for $l=1,2$ are characteristic polynomials by Lemma (36). Therefore $\mathrm{CH}^{j_{1}}(y / K)=\mathrm{CH}^{j_{2}}(y / M)$. Namely let $a_{J} \in Q\left(L \otimes_{K} M\right)$ be a coefficient of $\mathrm{CH}(y / K)$. Then $j_{1}\left(a_{J}\right)=j_{2}\left(a_{J}\right)$ in $Q\left(L \otimes_{K} M\right) \otimes_{L} Q\left(L \otimes_{K} M\right)$. It follows from Sublemma (25.5) that $a_{J}$ is in $L$. Now assume that $K$ is not necessarily algebraically closed. Let $\bar{K}$ be an algebraic closure of $K$. It follows from what we have proved $\mathrm{CH}(y / K)=\mathrm{CH}(y / \bar{K})$ is in $Q\left(L \otimes_{K} \bar{K}\right)\{Y\}$. As in the definition of $\mathrm{CH}(y / \bar{K})$ only a finite number of elements are involved, we may assume that there exists a finite algebraic extension $M \supset K$ such that $\mathrm{CH}(y \mid K)=\mathrm{Ch}(y$; $M / K$ ). We may further assume that the extension $M \supset K$ is Galois with Galois group $G$. Therefore $L \otimes_{K} M$ which is a field, is Galois over $L$ 
with Galois group $G$.

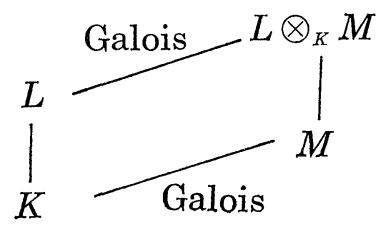

Let $g: M \rightarrow M$ be a $K$-automorphism. By the unicity of the minimal polynomial $\mathrm{Ch}(y ; M / K)$ is $G$-invariant or $\mathrm{CH}(y / K)=\mathrm{Ch}(y ; M / K)$ is in $L$.

Example (40). We can reformulate the definition of rational dependence and algebraic dependence in terms of characteristic polynomial. The extension $L=K\langle y\rangle \supset K$ depends rationally (resp. algebraically) on the initial conditions if and only if the rank of the absolute characteristic polynomial $\mathrm{CH}(y / K)$ is equal to $(1,0, \cdots)$ (resp. lower than $(0,1,0, \cdots)$ ).

Lemma (41) ([N1], Proposition)). Let $K \subset L$ be a differential field extension, which is regular and $F_{1}, F_{2} \subset L$ be differential subfields containing $K$. We assume that the fields $F_{1}$ and $F_{2}$ depends rationally on the initial conditions. Then the field $F$ generated by $F_{1}$ and $F_{2}$ depends rationally on the initial conditions.

Proof. First we assume that $K$ is algebraically closed. There exists differential field extensions $M_{1}$ and $M_{2}$ of $K$ such that $Q\left(F_{2} \otimes_{K} M_{1}\right)$ is generated over $M_{1}$ by constants and such that $Q\left(F_{1} \otimes_{K} M_{2}\right)$ is generated over $M_{2}$ by constants. Let $M=Q\left(M_{1} \otimes_{K} M_{2}\right)$. Then $Q\left(F_{i} \otimes_{K} M\right)=$ $Q\left(Q\left(F_{i} \otimes_{K} M_{i}\right) \otimes_{M_{i}} M\right)$ is generated over $M$ by constants since $Q\left(F_{1} \otimes_{K} M_{i}\right)$ is generated over $M_{i}$ by constants $(i=1,2)$. Hence $Q\left(F_{1} F_{2} \otimes_{K} M\right)=$ $Q\left(F_{1} \otimes_{K} M\right) Q\left(F_{2} \otimes_{K} M\right)\left(\subset Q\left(L \otimes_{K} M\right)\right)$ is generated over $M$ by constants. Now we treat the general case where $K$ is not necessarily algebraically closed. Let $\bar{K}$ be an algebraic closure of $K$. Then we can apply the argument above for $Q\left(L \otimes_{K} \bar{K}\right) \supset Q\left(F_{1} \otimes_{K} \bar{K}\right), Q\left(F_{2} \otimes_{K} \bar{K}\right) \supset \bar{K}$. The differential fields $Q\left(F_{1} \otimes_{K} \bar{K}\right)$ and $Q\left(F_{2} \otimes_{K} \bar{K}\right)$ depend rationally on the initial conditions. Since $Q\left(F_{1} \otimes_{K} \bar{K}\right) Q\left(F_{2} \otimes_{K} \bar{K}\right)=Q\left(F_{1} F_{2} \otimes_{K} \bar{K}\right), F_{1} F_{2}=F$ depends rationally on the initial conditions.

Theorem (42). There exists a differential intermediate field $L=K\langle y\rangle$ $\supset F \supset K$ satisfying the following conditions. 
(1) The extension $F \supset K$ depends rationally on the initial conditions.

(2) The minimal polynomial of $y$ over $F$ coincides with the absolute characteristic polynomial $\mathrm{CH}(y / K)$.

(3) Let $L \supset N \supset K$ be a differential intermediate field such that the extension $N \supset K$ depends rationally on the initial conditions. Then $N$ is a subfield of $F$.

The differential field $F$ is characterized by the conditions (1) and (2). $F$ is also characterized by the condition (3).

Proof. Let $M \supset K$ be a differential field extension such that the characteristic polynomial $\mathrm{Ch}(y ; M / K)$ gives the absolute characteristic polynomial $\mathrm{CH}(y / K)$.

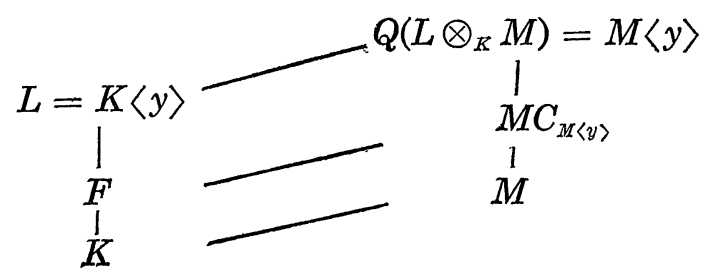

By Proposition (39), the coefficients of $\mathrm{CH}(y / K)$ is in $L$. Let $F$ be a differential subfield of $L$ generated over $K$ by the coefficients of $\mathrm{CH}(y / K)$. $Q\left(F \otimes_{K} M\right)$ is isomorphic to $F M$ in $M C_{M\langle y\rangle}$ and hence $F M=M C_{F M} ; F M$ is generated over $M$ by constants by Proposition (12). It follows from Lemma (33), $M C_{M\langle y\rangle}=F M$. Since $\mathrm{CH}(y / K)$ is the minimal polynomial of $y$ over $F M$ and since $\mathrm{CH}(y / K)$ is in $L\{Y\}, \mathrm{CH}(y / K)$ is the minimal polynomial of $y$ over $F$. The assertions (1) and (2) are proved. Let $L \supset N \supset K$ be an intermediate differential field which depends rationally on the initial conditions. Let us assume that $N$ is not contained in $F$. Then $L \supset F N$ $=F^{\prime} \supseteq F \supset K$ and the extension $F^{\prime} \supset K$ depends rationally on the initial conditions by Lemma (41). It follows from Lemma (34) there exists a differential field extension $M \supset K$ such that $\mathrm{Ch}(y ; M / K)=\mathrm{CH}(y / K)$ and such that $Q\left(F^{\prime} \otimes_{K} M\right)=F^{\prime} M\left(C_{Q\left(L \otimes_{K} M\right)}\right)$ is generated over $M$ by constants. It follows from Lemma (33) and the definition of $\mathrm{CH}(y / K)$, the inclusion $Q\left(F \otimes_{K} M\right)=F M \subset F^{\prime} M=Q\left(F^{\prime} \otimes_{K} M\right)$ should be an equality. Hence $F=F^{\prime}$, which is a contradiction and $N$ should be a subfield of $F$.

Now we are able to prove Theorem (31).

Proof of Theorem (31). Let $y=y\left(x_{0} ; z ; x\right)$ be the general solution in 
the usual analytic sense and let $K$ be a differential field generated over $\mathbb{C}$ by $R$. Let $L=K\langle y\rangle \supset K$. It follows from Example (40) that the rank of $\mathrm{CH}(y / K)$ is lower than $(0,1,0, \cdots)$. By Theorem (42), we can find a differential subfield $K \subset M \subset L$ such that the extension $M \supset K$ depends rationally on the initial conditions and such that $L$ is algebraic over $M$. We may assume that $K \supset \mathbb{Q}(x)$ with $x^{\prime}=1$ and then it follows from [K2], Chap. II, $\S 8$, Proposition 9 that $M=K\langle w\rangle$ for an appropriate element $w \in M$ and there exists a differential polynomial $G(W) \in K\{W\}$ such that $M\langle w\rangle$ is a generic solution of a differential equation $G(w)=0$. Since a solution of $F=0$ with general initial conditions is algebraic over a differential field generated over $K$ by a solution of $G=0$ with general initial conditions, it is obtained from $K$ by a finite iteration of the permissible operations (O), (P1), (P2), . , (P5) by Theorem (29).

Corollary (42.1). Let $L \supset K$ be a differential field extension which is regular and finitely generated as an abstract field extension. Then there exists an intermediate differential field $L \supset N \supset K$ satisfying the following conditions.

(i) The extension $N \supset K$ depends rationally on the initial conditions.

(ii) Let $L \supset M \supset K$ be a differential intermediate field such that the extension $M \supset K$ depends rationally on the initial conditions. Then $M$ is a subfield of $N$.

Proof. Let $x$ be a variable over $K$ and $\mathscr{K}=K(x)$ which is a differential over-field of $K$ if we define $\delta(x)=1$. Let $\mathscr{L}=Q\left(L \otimes_{K} K(x)\right)$. Then by [K2], Chap. II, $\S 8$, Proposition $9, \mathscr{L}$ is generated over $\mathscr{K}$ by a single element: $\mathscr{L}=\mathscr{K}\langle y\rangle$. We can apply Theorem (43): namely there exists a differential intermediate field $\mathscr{L} \supset \mathscr{N} \supset \mathscr{K}$ which depends rationally on the initial conditions and which is maximum among such subfields. Since the subfield $\mathscr{N}$ is unique, by the descent theory we can find a intermediate field $L \supset N \supset K$ such that $Q\left(N \otimes_{K} \mathscr{K}\right)=\mathscr{N}$ (cf. Weil [W], Theorem $4)$.

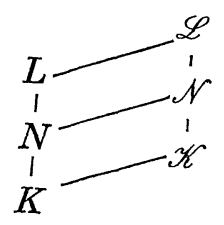


Since $L \cap \mathscr{N}=N, N$ is a differential subfield of $L$. The extension $N \supset K$ depends rationally on the initial conditions since the extension $\mathscr{N} \supset \mathscr{K}$ has this property. The intermediate field $L \supset N \supset K$ satisfies the condition (ii) for the same reason as above.

For the geometric meaning of the intermediate field $M$ see Proposition (49) in $\S 3$.

Remark (42.2). By Corollary (42.1), we can generalize Theorem (31) to a differential system.

\section{§3. Criterion of irreducibility for a second order differential equation free from moving singular points}

Let $R$ be a $\mathbb{C}$-algebra of holomorphic functions on a domain $D \subset \mathbb{C}$. Let $F\left(Y_{0}, Y_{1}\right) \in R\left[Y_{0}, Y_{1}\right]$ be an absolutely irreducible polynomial. We introduced the fibration $p: X \rightarrow D$ in $\S 1$. Replacing $D$ by a subdomain if necessary, we may assume that the fibres of $p$ are irreducible and $\partial F / \partial Y_{1}$ is not constantly equal to 0 on any fibre of $p$ ([U1]). For an algebraic differential equation $F\left(y, y^{\prime}\right)=0$, we can speak of fixed singular points, the singular points (branch points and transcendental singular points) of a solution $y\left(x_{0}, \bar{z}, x\right)$ independent of the initial conditions $\bar{z}$ at a fixed general point $x_{0}$. Other singular points of $y\left(x_{0}, \bar{z}, x\right)$ depending on the initial conditions $\bar{z}$ are not easy to describe. It is known that these singular points are branch points. We have the following classical theorem (cf. [F], Chap. IX and [M1]).

Theorem (43). The following conditions are equivalent.

(1) $F\left(x ; y, y^{\prime}\right)=0$ is free from movable branch points.

(2) For any point $x_{0} \in D$ different from the fixed singular points, there exists $a$ dense open set $V$ of $U=\left\{z \in X_{x_{0}} \mid \partial F / \partial Y_{1}\left(x_{0}, z\right) \neq 0\right\}$ and a subdomain $x_{0} \in D_{0} \subset D$ such that the function $y\left(x_{0} ; z ; x\right)$ in $(z, x)$ is meromorphic on $V \times D_{0}$.

(3) There exist a point $x_{0} \in D$ different from the fixed singular points and $a$ dense open set $V$ of $U=\left\{z \in X_{x_{0}} \mid \partial F / \partial Y_{1}\left(x_{0}, z\right) \neq 0\right\}$ and a subdomain $x_{0} \in D_{0} \subset D$ such that the function $y\left(x_{0} ; z ; x\right)$ in $(z, x)$ is meromorphic on $V \times D_{0}$.

(4) The general solution $y\left(x_{0} ; z ; x\right)$ of $F=0$ depends rationally on the initial conditions.

(5) Let $K$ be a differential field of meromorphic functions generated 
by $R$ and $\mathfrak{X}$ be the non-singular projective model of the algebraic function field $Q\left(K\left[Y_{0}, Y_{1}\right] / F\left(Y_{0}, Y_{1}\right)\left(Y_{0}, Y_{1}\right)\right)$ of one variable over $K$. Then for any point $p \in \mathfrak{X}$, the local ring $\mathcal{O}_{p}$ is closed under the derivation $\delta$.

For the second order algebraic differential equation the structure of singular points is more complicated. There are equations free from movable singular points whose general solution does not depend rationally on the initial conditions. Moreover there may be movable transcendental singular points. Let $F\left(Y_{0}, Y_{1}, Y_{2}\right) \in R\left[Y_{0}, Y_{1}, Y_{2}\right]$ be an absolutely irreducible polynomial. What the classical authors were looking for is the algebraic differential equation of second order satisfying the following condition.

Conditions (44). There exist a dense open set $D^{\prime}$ of $D$ satisfying the following the conditions.

(1) For any point $x_{0} \in D^{\prime}, X_{x_{0}}$ is reduced and irreducible and $\partial F / \partial Y_{2}\left(x_{0} ; z\right)$ is not constantly equal to 0 on $X_{x_{0}}$.

(2) For any point 2 points $x_{1}, x_{2} \in D^{\prime}$ and for any path $\Gamma$ in $D^{\prime}$ the correspondence $z \rightarrow\left(y\left(x_{1} ; z ; x_{2}\right), y^{\prime}\left(x_{1} ; z ; x_{2}\right) . y^{\prime \prime}\left(x_{1} ; z ; x_{2}\right)\right)$ induced by the analytic continuation along $\Gamma$ defines a biholomorphic map between dense open sets for the usual topology of $X_{x_{1}}$ and $X_{x_{2}}$.

Example (45). The first differential equation of Painlevé satisfies the conditions (44). In fact it is proved in Painlevé: Mémoire sur les équations différentielles dont l'intégrale général est uniforme, vol. 3, [P] that we can take (in the conditions (44)) $\mathbb{C}$ for $D^{\prime}$ and any point of $\mathbb{C}$ for $x_{0}$ (see also pp. 346-351 of [I]). Okamoto [O] clarified this fact in the modern langulage of foliation.

Definition (46). Let $L=K\langle y\rangle \supset K$ be a differential field extension with tr. d. $[L: K]=2$. If the rank of the absolute characteristic polynomial $\mathrm{CH}(y / K)$ is lower than $(0,0,1,0, \cdots)\left(j_{n}=0\right.$ except for $n=2$ and $j_{2}=1$ ), we say that the extension $L \supset K$ depends semi-transcendentally on the initial conditions. If the rank of the absolute characteristic polynomial $\mathrm{CH}(y / K)$ is higher than or equal to $(0,0,1,0, \cdots)\left(j_{n}=0\right.$ except for $n=2$ and $j_{2}=1$ ), we say that the extension $L \supset K$ depends essentially transcendentally on the initial conditions.

It follows from Theorem (42) that $L \supset K$ depends semi-transcendentally on the initial conditions if and only if there exists an intermediate differential field $L \supset F \supset K$ such that tr. d. $[L: F] \leq 1$ and such that the 
extension $F \supset K$ depends rationally on the initial conditions. Hence the definition that $L \supset K$ depends semi-transcendentally on the initial conditions is independent of the particular generator $y$ over $K$.

Let us study the differential equation (6) in $\S 1$. Let $R$ be a ring of holomorphic functions on a domain $D$ such that $F\left(Y_{0}, Y_{1}, \cdots, Y_{n}\right) \in$ $R\left[Y_{0}, Y_{1}, \cdots, Y_{n}\right]$. Let us assume that $R$ is a $\mathbb{C}$-algebra of finite type. We are interested in the case $n=2$ with $\partial F / \partial Y_{2} \neq 0$.

(47) $F\left(x ; y, y^{\prime}, y^{\prime \prime}\right)=0$.

Hence for the general solution $y$ of (47), we have tr. d. $[K\langle y\rangle: K]=2$, where $K$ is the differential field generated by $R$.

Definition (48). Let $y=y\left(x_{0} ; z ; x\right)$ be the general solution of (47). If the extension $K\langle y\rangle \supset K$ depends semi-transcendentally (resp. essentially transcendentally) on the initial conditions, we say that the general solution of (47) depends semi-transcendentally (resp. essentially transcendentally) on the initial conditions.

Remarks (48.1). Let $L=K\langle y\rangle$ and $J \supset K$ be a differential field extensions with tr. d. $[L: K]=2$. Then the extension $L \supset K$ depends semitranscendentally (resp. essentially transcendentally) on the initial conditions if and only if the extension $Q\left(L \otimes_{K} J\right) \supset J$ has the same property as $L \supset K$ (cf. Remarks (11.1) and (20.1)).

(48.2) By Theorem (42), the general solution $y$ of (47) depends semitranscendentally on the initial conditions if and only if there exists an

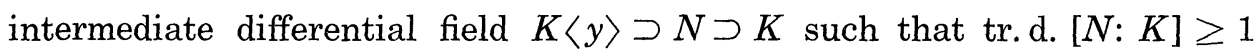
and such that $N \supset K$ depends rationally on the initial conditions. Geometrically, this is equivalent to saying that the transformation between two initial conditions or two fibres of $p: X \rightarrow D$ induced by solutions as explained in the sentence following Definition (7), maps a non-constant rational function on a fibre of $p$ to a (non-constant) algebraic function on the other fibre, which is the original intuitive definition introduced by Painlevé and from which the word semi-transcendental is motivated (see Proposition (49) below). We notice that his definition is different from ours. According to our Definition (48), if the general solution depends algebraically on the initial conditions, then it depends semitranscendentally on the initial conditions, whereas Painleve excludes this type of equations from the definition of semi-transcendental dependence. 
The following Proposition explains the geometric meaning of the semi-transcendental dependence on the initial conditions.

Proposition (49). We assume that the ring $R$ is of finite type over $\mathbb{C}$. The following conditions for the differential equation (47) are equivalent.

(i) The general solution depends semi-transcendentally on the initial conditions.

(ii) For a general point $x_{0} \in D$, there exist a $\mathbb{C}$-algebra $R^{\prime}$ containing $R$ and of finite type over $\mathbb{C}$ consisting of holomorphic functions on a subdomain $x_{0} \in D^{\prime}$ and an algebraic subvariety $\mathscr{Z} \subset X_{x_{0}} \times \mathscr{X}_{R^{\prime}}=\left(X_{x_{0}} \otimes_{\mathrm{C}} R^{\prime}\right) \times_{R^{\prime}}$ $\left(\mathscr{X} \otimes_{R} R^{\prime}\right)$ such that $(0) y\left(x_{0} ; z ; x\right)$ is regular for $(z, x) \in V \times D^{\prime}$ (where $V$ is an appropriate open set of $\left.U=\left\{z \in X_{x_{0}} \mid \partial F / \partial Y^{\prime \prime}\left(x_{0} ; z\right) \neq 0\right)\right\}$, (1) $\left(z ; y\left(x_{0} ; z\right.\right.$; $\left.x), y^{\prime}\left(x_{0} ; z ; x\right), \quad Y^{\prime \prime}\left(x_{0} ; z ; x\right)\right)$ is in $\mathscr{Z} \mid D^{\prime}$ for $(z, x) \in V \times D^{\prime}$ and such that (2) the dimension of the generic fibre of $\mathscr{Z} \rightarrow \operatorname{Spec} R^{\prime}$ is at most 3 (cf. $\S 1$, $(6.0))$.

Proof. We content ourselves with giving an outline of the proof since the proof is done in the same spirit as in the proof of the equivalence of 2 definitions of rational dependence on the initial conditions (cf. $\S 1$ ). Let $K$ be the differential field generated over $\mathbb{C}$ by $R$. If the condition (i) is satisfied, then there exists a differential field extension $M \supset K$ such that tr. d. $\left[C_{Q\left(K\langle y\rangle \otimes_{K} M\right.}: C_{M}\right] \geq 1$, where $y$ denotes the general solution $y\left(x_{0} ; z ; x\right)$. The argument in $\S 1$ allows us to assume that $M$ consists of meromorphic functions over a subdomain $D^{\prime}$ of $D$. Now we can identify $K\langle y\rangle \otimes_{K} M$ with a ring of meromorphic functions over $D^{\prime} \times V$ for an open set $V$ of $X_{x_{0}}$. Since tr.d. $\left[C_{Q\left(K\langle y\rangle \otimes_{K} M\right)}: C_{K}\right] \geq 1$, there exists a constant $0 \neq c \in K\langle y\rangle \otimes_{K} M=M\langle y\rangle$ which is transcendental over $M$. Let $c=A / B\left(y, y^{\prime}, y^{\prime \prime}\right)$ with $A, B \in M\left[y, y^{\prime}, y^{\prime \prime}\right]$. Then the argument of $\S 1$ shows that perhaps changing the reference point $x_{0}$ we may assume $c=C / D\left(z_{0}, z_{1}, z_{2}\right)$ with $C, D \in M\left[z_{0}, z_{1}, z_{2}\right]$. This shows that tr.d. $\left[M\left(y, y^{\prime}, y^{\prime \prime}, z_{0}, z_{1}, z_{2}\right): M\right] \leq 3$. Since there involved only finitely many elements, we can find a ring $R^{\prime}$ satisfying the condition (ii). Conversely now let us assume that the condition (ii) is satisfied. Then there exists a differential field $M$ of meromorphic functions over a subdomain $D^{\prime}$ such that tr. d. $\left[M\left(y, y^{\prime}, y^{\prime \prime}, z_{0}, z_{1}, z_{2}\right): M\right] \leq 3$. If tr. d. $\left[C_{M\left(y, y^{\prime}, y^{\prime \prime}\right)}: C_{M}\right]=0$, then $C_{M\left(y, y^{\prime}, y^{\prime \prime}\right)}=C_{M}=\mathbb{C}$. By [K2] Chap. II, 1, Corollary $1 M\left(y, y^{\prime}, y^{\prime \prime}, z_{0}, z_{1}, z_{2}\right)$ $\simeq M\left(y, y^{\prime}, y^{\prime \prime}\right) \otimes_{\mathrm{C}} \mathbb{C}\left(z_{0}, z_{1}, z_{2}\right)$ and hence tr. d. $\left[M\left(y, y^{\prime}, y^{\prime \prime}, z_{0}, z_{1}, z_{2}\right): M\right]=4$. Therefore tr. d. $\left[C_{M\left(y, y^{\prime}, y^{\prime \prime}\right)}: C_{M}\right] \geq 1$ and the condition (1) is satisfied. 
The same argument proves the following generalization of Proposition (49) which explains the geometric meaning of the intermediate differential field of Theorem (42).

Proposition (49.1). Let $K$ be the quotient differential field of $R$ and $L \supset K$ be a generic solution of (6) over $K$ (hence $L \supset K$ is $K$-isomorphic to $K\left\langle y\left(x_{0} ; z ; x\right)\right\rangle$ for a general point $\left.x_{0}\right)$. Let $L \supset M \supset K$ be the intermediate differential field of Theorem (42). Then the following conditions are equivalent.

(i) tr.d. $[M: K]=d$.

(ii) For a general point $x_{0}$, there exist a $\mathbb{C}$-algebra $R^{\prime}$ containing $R$ and of finite type over $\mathbb{C}$ consisting of holomorphic functions on a subdomain $x_{0} \in D^{\prime}$ and an algebraic subvariety $\mathscr{Z} \subset X_{x_{0}} \times \mathscr{X}=\left(X_{x_{0}} \otimes_{c} R^{\prime}\right) \times_{R^{\prime}}$ $\left(\mathscr{X} \otimes_{R} R^{\prime}\right)$ such that (1) $\left(z ; y\left(x_{0} ; z ; x\right), y^{\prime}\left(x_{0} ; z ; x\right), \cdots, y^{(n)}\left(x_{0} ; z ; x\right)\right)$ is in $\mathscr{Z}$ for $(z, x) \in V \times D^{\prime}$ (where $V$ is an appropriate open set of $U=z \in X_{x_{0}} \mid \partial F / \partial Y^{(n)}\left(x_{0} ; z\right)$ $\neq 0\})$ and (2) the dimension of the generic fibre of $\mathscr{Z} \rightarrow \mathrm{Spec} R^{\prime}$ is equal to $2 n-d$. If there exist an algebra $R^{\prime \prime}$ containing $R$ and of finite type over $\mathbb{C}$ consisting of holomorphic functions on a subdomain $x_{0} \in D^{\prime \prime}$ and an algebraic subvariety $\mathscr{Z}^{\prime} \subset X_{x_{0}} \times \mathscr{X} \otimes_{R} R^{\prime \prime}=\left(X_{x_{0}} \otimes_{\mathrm{c}} R^{\prime \prime}\right) \times_{R}\left(\mathscr{X} \otimes_{R} R^{\prime \prime}\right)$ such that (1) $\left(z ; y\left(x ; z_{0} ; x\right), y^{\prime}\left(x ; z_{0} ; x\right), \cdots, y^{(n)}\left(x ; z_{0} ; x\right)\right)$ is in $\mathscr{Z}^{\prime}$ for $(z, x) \in V^{\prime} \times D^{\prime \prime}$ (where $V^{\prime}$ is an appropriate open set of $U=\left\{z \in X_{x_{0}} \mid \partial F / \partial Y^{(n)}\left(x_{0} ; z\right) \neq 0\right\}$ ), then the dimension of the generic fibre of $\mathscr{Z}^{\prime} \rightarrow \operatorname{Spec} R^{\prime \prime}$ is at least $2 n-d$.

We can also prove the same result for not necessarily simple extensions but we do not touch here the proof since it is the same.

THEOREM (50). Let $K_{0}$ be a differential field of meromorphic functions on a domain $D$ which is differentially finitely generated over $\mathbb{Q}$ (hence the field $K_{0}$ consists of denumerable number of elements). Let $K$ be a differential field generated by $K_{0}$ over $\mathbb{C}$. We assume that the coefficients of the differential equation (47) are in $K_{0}$. If the differential equation (47) satisfies the conditions (44), then the following conditions are equivalent.

(1) Any holomorphic solution of $F=0$ with general initial conditions at a general point is obtained from $K$ by a finite iteration of the permissible operations $(\mathrm{O}),(\mathrm{P} 1),(\mathrm{P} 2), \cdots,(\mathrm{P} 6)$.

$\left(1^{\prime}\right)$ Let $y$ be a holomorphic solution on a subdomain of $D$ such that tr. d. $\left[K_{0}\langle y\rangle ; K_{0}\right]=2$ : namely $y$ is a generic solution of $F=0$ over $K_{0}$. Then $y$ is obtained from $K_{0}$ by a finite iteration of the permissible operations (O), (P1), (P2), .., (P6). 
(2) Any holomorphic solution of $F=0$ with a general initial conditions at a general point is obtained from $K$ by a finite iteration of the permissible operations (O), (P1), (P2), , , (P5).

(2') Let $y$ be a holomorphic solution on a subdomain of $D$ such that tr. d. $\left[K_{0}\langle y\rangle: K_{0}\right]=2$ : namely $y$ is a generic solution of $F=0$ over $K_{0}$. Then $y$ is obtained from $K_{0}$ by a finite iteration of the permissible opera-

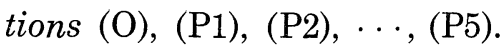

(3) The general solution $y\left(x_{0} ; z ; x\right)$ of $F=0$ depends semi-transcendentally on the initial conditions.

Proof. Let us show that the condition (1) implies (1'). In fact let $x_{0} \in D$ be a general point and let $y\left(x_{0} ; z ; x\right)$ be the general solution. Let $R_{0}=\left\{f \in K_{0} \mid f\right.$ is regular at $\left.x_{0}\right\}$ and $\mathfrak{m}_{0}=\left\{f \in R_{0} \mid f\left(x_{0}\right)=0\right\}$. Then a subset $R_{0} / \mathfrak{m}_{0} \subset \mathbb{C}$ is countable. Therefore we can find a $\mathbb{C}$-valued point $\bar{z}$ of a Zariski open set of $X_{x_{0}}$ such that $y\left(x_{0} ; \bar{z} ; x\right)$ and $y^{\prime}\left(x_{0} ; \bar{z} ; x\right)$ are algebraically independent over $R_{0} / \mathfrak{m}_{0}$. This implies that $y\left(x_{0} ; \bar{z} ; x\right)$ and $y^{\prime}\left(x_{0} ; \bar{z} ; x\right)$ are algebraically independent over $K_{0}$. Hence we can find a generic solution $y\left(x_{0} ; \bar{z} ; x\right)$ of $F=0$ over $K_{0}$ which is obtained from $K$ by a finite iteration of the permissible operations $(\mathrm{O}),(\mathrm{P} 1),(\mathrm{P} 2), \cdots,(\mathrm{P} 6)$. Then $y(x ; \bar{z} ; x)$ is a generic solution of $F=0$ over $K_{0}$. Since the extension $K \supset K_{0}$ is generated by constants and hence each element of $K$ is obtained from $K_{0}$ by finite iteration of the permissible operations (P1) and (P3) and since $y\left(x_{0} ; \bar{z} ; x\right)$ is obtained from $K$ by a finite iteration of the permissible operations $(\mathrm{O}),(\mathrm{P} 1),(\mathrm{P} 2), \cdots,(\mathrm{P} 6)$, therefore $y\left(x_{0} ; \bar{z} ; x\right)$ is obtained from $K_{0}$ by a finite iteration of the permissible operations (O), (P1), (P2), . , (P6). Since any generic solution of $F=0$ over $K_{0}$ determines a differential field extension of $K_{0}$ isomorphic to $K_{0}\left\langle y\left(x_{0} ; \bar{z} ; x\right)\right\rangle \supset K_{0}$, the condition $\left(1^{\prime}\right)$ is satisfied. The same argument shows that the condition (2) implies (2'). The condition (2) implies (1) since in (1) we have one more permissible operations. For the same reason the condition $\left(2^{\prime}\right)$ implies $\left(1^{\prime}\right)$. Let us show that the condition (3) implies (2). Let us assume the condition (3). If the condition (3) is satisfied, then by Theorem (42) there exists a differential intermediate field $K \subset M \subset L=K\left\langle y\left(x_{0} ; z ; x\right)\right\rangle$ such that the extension $M \supset K$ depends rationally on the initial conditions and such that the rank of the minimal polynomial of $y$ over $M$ is lower than $(0,0,1,0, \cdots)$ or equivalently tr. d. $[M\langle y\rangle: M] \leq 1$. We may assume tr. d. $[M\langle y\rangle: M]=1$. For otherwise, the condition (3) implies (2) by Theorem (31). Namely we may assume that $y$ satisfies an algebraic dif- 
ferential equation $G\left(y, y^{\prime}\right)=0$ with coefficients in $M$ such that $G(Y)$ is the absolute characteristic polynomial of $y$. The differential field $M$ is generated over $K$ by the coefficients of $G$. By [K2], Chap. II, $\S 8$, Proposition 9 , the differential field $M$ is generated over $K$ by a single element $u ; M=k\langle u\rangle$ (notice that we may assume $\mathbb{C}(x) \subset K$ so that the condition of the proposition in [K2] is satisfied). Therefore by Theorem (30) there exists a differential field $N$ whose elements are obtained from $K$ by a finite iteration of the permissible operations (O), (P1), (P2), . , (P5) such that $Q\left(M \otimes_{K} N\right)$ is generated over $N$ by constants $c_{1}, c_{2}, \cdots, c_{m}$.

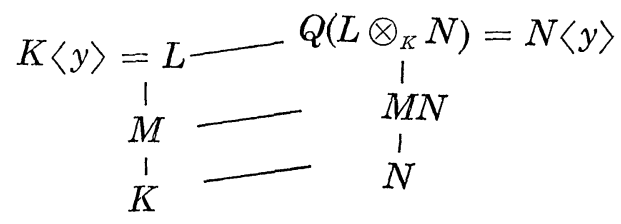

Then $y=y\left(x_{0} ; z ; x\right)$ is a generic solution of $G=0$ over $M N$ by definition. The differential equation $G=0$ is parametrized by $c_{1}, c_{2}, \cdots, c_{m} ; G=$ $G\left(c_{1}, c_{2}, \cdots, c_{m} ; x ; Y ; Y_{1}\right)$. We may assume by Theorem (29) that there exist a subdomain $D_{0} \subset D^{\prime}$ and a dense open set $V \subset\left\{z \in X_{x_{0}} \mid \partial F / \partial Y_{2} \neq 0\right\}$ such that the elements of $N$ are meromorphic on $D_{0}$ and such that $N\langle y\rangle$ and hence $K\langle y\rangle$ are in the field of the meromorphic functions on $V \times D_{0}$. We noticed there that the choice of $D_{0}$ is free. By [K2], Chap. II, $\S 1$, Corollary 1 , we have $M N=Q\left(\mathbb{C}\left[c_{1}, c_{2}, \cdots, c_{m}\right] \otimes_{\mathbb{C}} N\right)$. Let $Z=\operatorname{Spec} \mathbb{C}\left[c_{1}\right.$, $\left.c_{2}, \cdots, c_{m}\right]$. Let $\mathscr{V}$ be the complete non-singular model of the algebraic function field $L N$ of one variable over $M N$. The $N$-algebraic variety $Z \otimes_{\mathrm{c}} N$ parametrizes the extensions of $N$ or curves whose generic fibre is $\mathscr{V} / M N$. There exists an $N$-algebraic variety $\mathscr{V}_{0}$ smooth and projective over a Zariski open set $Z_{0}$ of $Z \otimes_{\mathrm{C}} N=\operatorname{Spec} N\left[c_{1}, c_{2}, \cdots, c_{m}\right]$ such that $\mathscr{V} / M N$ is the generic fibre of $\mathscr{V}_{0} / Z_{0}$. Let us denote the $N$-morphism $\mathscr{V}_{0}$ $\rightarrow Z_{N}$ by $f$. Let $x_{0}^{\prime}$ be a general point of $D_{0}$ so that the coefficients of the rational map $f: \mathscr{V}_{0} \rightarrow z_{N}$ is regular at $x_{0}^{\prime}$ and so that we have a dominant $\mathbb{C}$-morphism $f^{\prime}: \mathscr{V}^{\prime}=\mathscr{V}_{0 x_{0}^{\prime}} \rightarrow Z$ by reduction at $x_{0}$ of $f$. Since $\mathscr{V}^{\prime}$ is $\mathbb{C}$-birational to $X_{x_{0}^{\prime}}$, we have a dominant $\mathbb{C}$-rational map $h: X_{x_{0}^{\prime}} \cdots \rightarrow Z$. It follows from Condition (44) $y\left(x_{0} ; z ; x\right)=y\left(x_{0}^{\prime} ; z ; x\right)$ and there exists a dense open set $V^{\prime} \subset X_{x_{0}^{\prime}}$ such that the rational map $h$ is regular on $V^{\prime}$ and such that the function $y\left(X_{0}^{\prime} ; z^{\prime} ; x\right)$ in $\left(z^{\prime}, x\right)$ is regular on $V^{\prime} \times D_{0}$. Since $h$ is dominant, $h\left(V^{\prime}\right)$ contains a dense open set $\tilde{Z}$ of $Z$ for the usual 
topology. If $d=\left(d_{1}, d_{2}, \cdots, d_{m}\right) \in Z$ is in $\tilde{Z}$, then the differential equation $G\left(d_{1}, d_{2}, \cdots, d_{m} ; x ; y, y^{\prime}\right)=0$ whose coefficients are in $N$, satisfies the condition (3) of Theorem (43) for the reference point $x_{0}^{\prime}$ since $y\left(x_{0}^{\prime} ; z^{\prime} ; x\right)$ with $z^{\prime} \in V^{\prime}, h\left(z^{\prime}\right)=d$ is the general solution of $G\left(d ; x ; y, y^{\prime}\right)=0$. Therefore the condition (4) of Theorem (43) is satisfied for $G\left(d ; x ; y, y^{\prime}\right)=0$ and hence any solution of $G\left(d ; x ; y, y^{\prime}\right)=0$ with general initial conditions at $x_{0}^{\prime}$ is obtained from $N$ by a finite iteration of the permissible operations (O), (P1), (P2), ‥, (P5) by Theorem (30). Since the field $N$ is constructed by finite iterations of the permissible operations (O), (P1), (P2), . , (P5) from $K$ by Theorem (30), The condition (2) of the theorem is thus satisfied.

It remains to show that the condition (1') implies the condition (3). The field $K_{0}$ consists of countably many elements. As we have seen at the begining of the proof, we can find a solution $y(x)$ with general initial condition at $x_{0}$ such that tr. d. $\left[K_{0}\langle y(x)\rangle ; K_{0}\right]=2$. It follows from the condition ( $\left.1^{\prime}\right)$ and Theorems (2) and (3) that we can find a chain of differential fields

$$
K=M_{0} \subset M_{1} \subset \cdots \subset M_{m} \quad \text { with } y=y(x) \in M_{m},
$$

which are differential subfields of the field of the meromorphic functions over a subdomain of $D$ such that the field $M_{i}$ is finitely generated over $M_{i-1}$ and such that the extension $M_{i} \supset M_{i-1}$ satisfies one of the following conditions for $1 \leq i \leq m$;

(50.1) tr. d. $\left[M_{i}: M_{i-1}\right]=1$ and $M_{i}=M_{i-1}\left\langle z_{i}\right\rangle$ for an appropriate element $z_{i} \in M_{i}$;

(50.2) There exists an algebraic group $G_{i}$ defined over $\mathbb{C}$ and a $M_{i}$ valued point $z_{i}$ of $G_{i} \otimes_{\mathrm{C}} M_{i}$ such that $M_{i-1}\left(z_{i}\right)=M_{i}$ and $l \delta\left(z_{i}\right) \in \mathfrak{g}_{i} \otimes_{\mathrm{C}} M_{i}$ lies in $\mathfrak{g}_{i} \otimes_{\mathrm{c}} M_{i-1}$, where $\mathrm{g}_{i}$ is the Lie algebra of $G_{i}$.

The first case occurs in the operation (P6) and the second case appears in the other operations by Theorem (2). We can do this starting from the smaller field $K_{0}$. Namely we can find a chain of differential fields $K_{0} \subset K_{1} \subset \cdots \subset K_{n}$ with $y(x) \in K_{n}$ which are differential subfields of the field of the meromorphic functions over a subdomain of $D$ such that the field $K_{i}$ is finitely generated over $K_{i-1}$ and such that the extension $K_{i} \supset$ $K_{i-1}$ satisfies one of the following conditions;

(51. i) $K_{i}$ is generated over $K_{i-1}$ by constants;

(51.ii) tr.d. $\left[K_{i}: K_{i-1}\right]=1$ and $K_{i}=K_{i-1}\left\langle z_{i}\right\rangle$ for an appropriate element $z_{i} \in K_{i}$ 
(51.iii) There exist an algebraic group $H_{i}$ defined over the field of the constants $C_{i-1}$ of $K_{i-1}$ and a $K_{i}$-valued point $z_{i}$ of $H_{i-1} \otimes_{C_{i-1}} K_{i-1}$ such that $K_{i-1}\left(z_{i}\right)=K_{i}$ and $l \delta\left(z_{i}\right) \in \mathfrak{h}_{i} \otimes_{C_{i}} K_{i}$ lies in $\mathfrak{h}_{i} \otimes_{C_{i-1}} K_{i-1}$, where $\mathfrak{h}_{i}$ is the Lie algebra of $H_{i}$.

(52) Let us assume first that there is an index $i$ such that tr.d. $\left[K_{i-1}\langle y\rangle ; K_{i-1}\right]=2$ and tr. d. $\left[K_{i}\langle y\rangle: K_{i}\right]=0$. Since tr. d. $\left[K_{i-1}\langle y\rangle: K_{i-1}\right]$ $=2, y$ is a generic solution of $F=0$ over $K_{i-1}$ by [K2], Chap. IV, $\S 14$, Theorem $5 ; K_{i-1}\langle y\rangle \simeq K_{i-1}\{Y\} / \mathfrak{p}(F)$ where $\mathfrak{p}(F)$ is the differential ideal of the general solution of $F=0$ over $K_{i-1}$.

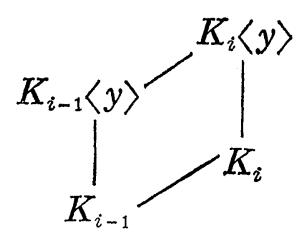

Subcase (54). The extension $K_{i} \supset K_{i-1}$ is of type (51.i).

If it is of type (51.i), putting $r=\operatorname{tr}$.d. $\left[K_{i}: K_{i-1}\right]$ we have tr.d. [ $C_{k_{i}}$ : $\left.C_{K_{i-1}}\right]=r$ and tr. d. $\left[C_{K_{i}\langle y(x)\rangle}: C_{K_{i}}\right] \leq \operatorname{tr}$.d. $\left[K_{i}\langle y(x)\rangle: K_{i}\right]=0$ by [K2] Chap. II, $\S 1$, Corollary 1. Hence tr.d. $\left[C_{K_{i\langle y(x)\rangle}}: C_{K_{i-1}}\right]=r$. Similarly we have tr.d. $\left[K_{i}\left\langle y\langle(x)\rangle: K_{i-1}\right]=r\right.$. Therefore tr. d. $\left[C_{K_{i-1}\langle y(x)\rangle}: C_{K_{i-1}}\right]=\operatorname{tr}$.d. $\left[C_{K_{i}\langle y(x)\rangle}:\right.$ $\left.C_{K_{i-1}}\right]-\operatorname{tr}$. d. $\left[C_{K_{i}\langle y(x)\rangle}: C_{K_{i-1}\langle y(x)\rangle}\right] \geq \operatorname{tr}$. d. $\left[C_{K_{i\langle y(x)\rangle}}: C_{K_{i-1}}\right]-\operatorname{tr}$.d. $\left[K_{i}\langle y(x)\rangle:\right.$ $\left.K_{i-1}\langle y(x)\rangle\right]$ by [K2], Chap. II, $\S 1$, Corollary 1. tr. d. $\left[C_{K_{i}\langle y(x)\rangle}: C_{K_{i-1}}\right]$ - tr. d. $\left[K_{i}\left\langle y(x): K_{i-1}\langle y(x)\rangle\right]=r-\right.$ tr. d. $\left[K_{i}\langle y(x)\rangle: K_{i-1}\langle y(x)\rangle\right]=$ tr. d. $\left[K_{i}\langle y(x)\rangle: K_{i-1}\right]-$ tr. d. $\left[K_{i}\langle y(x)\rangle: K_{i-1}\langle y(x)\rangle\right]=$ tr. d. $\left[K_{i-1}\langle y(x)\rangle: K_{i-1}\right]=2$. Therefore $2=\operatorname{tr}$.d. $\left[K_{i-1}\langle y(x)\rangle: K_{i-1}\right] \geq \operatorname{tr}$.d. $\left[C_{K_{i-1}\langle y(x)\rangle}: C_{K_{i-1}}\right] \geq 2$ and hence

$$
\text { tr. d. }\left[C_{K_{i-1}\langle y(x)\rangle}: C_{K_{i-1}}\right]=2 \text {. }
$$

We can find a subdomain $D^{\prime}$ of $D$ such that $K_{i-1} \subset K\left(D^{\prime}\right)$. By [K2], Chap. IV, § 14, Theorem 5, we have $K_{i-1}\langle y(x)\rangle \simeq Q\left(K_{i-1}\{Y\} / \mathfrak{p}(F)\right)$ and hence $Q\left(K_{i-1}\langle y(x)\rangle \otimes_{K_{i-1}} K\left(D^{\prime}\right)\right) \simeq K\left(D^{\prime}\right)\{Y\} / \mathfrak{P}(F)$, where $\mathfrak{p}(F)$ (resp. $\mathfrak{P}(F)$ ) is the prime differential ideal of the general solutions of $F=0$ in $K_{i}\{Y\}$ (resp. in $\left.K\left(D^{\prime}\right)\{Y\}\right)$. Since the rank of the minimal polynomial of $y(x)$ over $K_{i-1} C_{K_{i-1}\langle y(x)\rangle} \leq(0,1,0, \cdots)$ by $(55)$, the general solution of $F=0$ depends algebraically on the initial conditions and the condition (3) is satisfied since $y(x)$ is a generic solution $F=0$ over $K_{i-1}$ (cf. Remark (48.1)).

Subcase (56). The extension $K_{i} \supset K_{i-1}$ is of type (51.iii). 
Replacing $K_{i-1}$ by its algebraic closure in $K_{i}\langle y(x)\rangle$, we may assume that the extension $K_{i}\langle y(x)\rangle \supset K_{i-1}$ is regular. Tensoring $K_{i}$ over $K_{i-1}$ with (53), we get

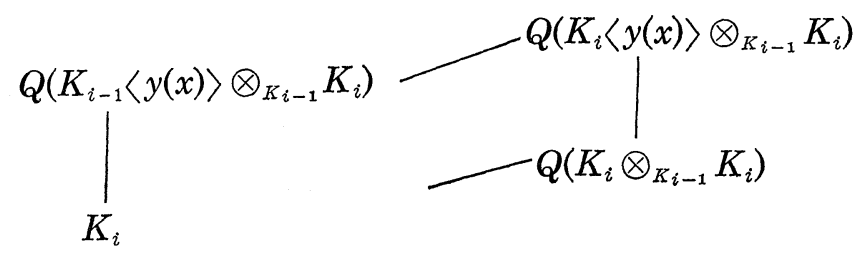

Since $Q\left(K_{i} \otimes_{K_{i-1}} K_{i}\right)$ is generated over $\varphi_{2}\left(K_{i}\right)$, which we denote also by $K_{i}$, by constants by Proposition (5) $\left(\varphi_{2}: K_{i} \rightarrow K_{i} \otimes_{K_{i-1}} K_{i}\right.$ being defined by $\left.\varphi_{2}(a)=1 \otimes a\right)$. Since tr. d. $\left[Q\left(K_{i-1}\langle y(x)\rangle \otimes_{K_{i-1}} K_{i}\right): K_{i}\right]=2, \quad Q\left(K_{i-1}\langle y(x)\rangle\right.$ $\left.\otimes_{K_{i-1}} K_{i}\right) \supset K_{i}$ is a generic solution of $F=0$ over $K_{i}$ and we conclude as in the above case that the general solution of $F=0$ depends algebraically on the initial conditions. Under the assumption (52), we have tr. d. $\left[K_{i}: K_{i-1}\right] \geq 2$ and hence an extension of type (51.ii) does not appear. Thus the case (52) is finished.

(58) Let us now assume that there exists an index $i$ such that tr. d. $\left[K_{i-1}\langle y(x)\rangle: K_{i-1}\right]=2$ and tr. d. $\left[K_{i}\langle y(x)\rangle: K_{i}\right]=1$.

Subcase (59). The extension $K_{i} \supset K_{i-1}$ is of type (51.i).

Let us put $r=\operatorname{tr}$.d. $\left[K_{i}: K_{i-1}\right]$. Since tr.d. $\left[K_{i}\langle y(x)\rangle: K_{i-1}\langle y(x)\rangle\right]=$ tr. d. $\left[K_{i}\langle y(x)\rangle: K_{i-1}\right]-$ tr. d. $\left[K_{i-1}\langle y(x)\rangle: K_{i-1}\right]=$ (tr. d. $\left[K_{i}\langle y(x)\rangle: K_{i}\right]+$ tr. d. $\left.\left[K_{i}: K_{i-1}\right]\right)-2=r-1$. It follows from [K2], Chap. II, $\S 1$, Corollary 1, tr.d. $\left[C_{K_{i}\langle y(x)\rangle}: C_{K_{i-1}\langle y(x)\rangle}\right] \leq r-1$. As tr.d. $\left[C_{K_{i}\langle y(x)\rangle}: C_{K_{i-1}}\right] \geq \operatorname{tr.d.}\left[C_{K_{i}}: C_{K_{i-1}}\right]$ $=r$ tr.d. $\left[C_{K_{i-1}\langle y(x)\rangle}: C_{K_{i-1}}\right]=\operatorname{tr} . \mathrm{d} .\left[C_{K_{i}\langle y(x)\rangle}: C_{K_{i-1}}\right]-\operatorname{tr} . \mathrm{d} .\left[C_{K_{i}\langle y(x)\rangle}: C_{K_{i-1}\langle y(x)\rangle}\right]$ $\geq r-(r-1) \geq 1$. Therefore the characteristic polynomial of $y(x)$ is lower than $(0,0,1,0, \cdots)$. Since tr. d. $\left[K_{i-1}\langle y(x)\rangle: K_{i-1}\right]=2, y(x)$ is a generic solution of $F=0$ over $K_{i-1}$. Therefore the rank of the absolute minimal polynomial of the general solution $y\left(x_{0} ; z ; x\right)$ is lower than $(0,0$, $1,0, \cdots)$ and the condition (3) is satisfied (cf. Remark (20.1)).

Subcase (60). The extension $K_{i} \supset K_{i-1}$ is of type (51.iii).

Replacing $K_{i-1}$ by its algebraic closure in $K_{i}$, we may assume that the extension $K_{i} \supset K_{i-1}$ is regular. Tensoring $K_{i}$ over $K_{i-1}$ with (53), we get (57). Since $Q\left(K_{i} \otimes_{K_{i-1}} K_{i}\right)$ is generated over $\varphi_{2}\left(K_{i}\right)$ by constants by Proposition (5) $\left(\varphi_{2}: K_{i} \rightarrow K_{i} \otimes_{K_{i-1}} K_{i}\right.$ being defined by $\varphi_{2}(a)=1 \otimes a$ for $\left.a \in K_{i}\right)$, we conclude as in subcase (59) that the general solution $y\left(x_{0} ; z ; x\right)$ 
depends semi-transcendentally on the initial conditions.

Subcase (61). The extension $K_{i} \supset K_{i-1}$ is of type (51.ii). As in the preceding case (60), we may assume that $K_{i-1}$ is algebraically closed in $K_{i}\langle y(x)\rangle$ so that the extension $K_{i}\langle y(x)\rangle \supset K_{i-1}$ is regular. Replacing $D$ by a subdomain if necessary, we may further assume that $K_{i-1} \subset K(D)$. Let us put $K_{i-1}=\mathscr{K}_{1}, K_{i-1}\langle y(x)\rangle=\mathscr{K}_{2}, K_{i}=\mathscr{K}_{3}$, and $K_{i}\langle y(x)\rangle=\mathscr{K}_{4}$ to simplify the notation. Since tr.d. $\left[\mathscr{K}_{3}: \mathscr{K}_{1}\right]=1$, tr.d. $\left[\mathscr{K}_{4}: \mathscr{K}_{3}\right]=1$ and since tr. d. $\left[\mathscr{K}_{2}: \mathscr{K}_{1}\right]=2$, hence tr. d. $\left[\mathscr{K}_{4}: \mathscr{K}_{2}\right]=\operatorname{tr}$.d. $\left[\mathscr{K}_{4}: \mathscr{K}_{1}\right]-\operatorname{tr}$. d. $\left[\mathscr{K}_{2}:\right.$ $\left.\mathscr{K}_{1}\right]=\operatorname{tr}$. d. $\left[\mathscr{K}_{3}: \mathscr{K}_{1}\right]+$ tr. d. $\left[\mathscr{K}_{4}: \mathscr{K}_{3}\right]-2=0 . \quad$ Namely $\mathscr{K}_{4}$ is algebraic over $\mathscr{K}_{2}$. Let $\mathscr{K}_{4}^{\prime}$ be the Galois closure of $\mathscr{K}_{4} \supset \mathscr{K}_{2}$. We denote by $G$ the Galois group of $\mathscr{K}_{4}^{\prime} \supset \mathscr{K}_{2}$. We show that the extension $\mathscr{K}_{3} \supset \mathscr{K}_{1}$ depends algebraically on the initial conditions. Since tr.d. $\left[\mathscr{K}_{2}: \mathscr{K}_{1}\right]=2$, the extension $\mathscr{K}_{2} \supset \mathscr{K}_{1}$ is a generic solution of $F=0$ over $\mathscr{K}_{1}$ and hence the differential field $\mathscr{K}_{2}$ is isomorphic to $\mathscr{L}_{2}=\mathscr{K}_{1}\left\langle y\left(x_{0} ; z ; x\right)\right\rangle$ over $\mathscr{K}_{1}$, where $y\left(x_{0} ; z ; x\right)$ is the general solution of $F=0\left(x_{0}\right.$ being a general point of $D$ ). It follows from the hypothesis that there exists a dense open set $V$ of $U=\left\{z \in X_{0} \mid \partial F / \partial Y_{2}\left(x_{0} ; z\right) \neq 0\right\} \subset X_{0}=\left\{z \in \mathbb{C}^{3} \mid F\left(x_{0} ; z\right)=0\right\}$ such that $y\left(x_{0} ; z ; x\right)$ is meromorphic on $D \times V$. Therefore we can find an extension $\mathscr{L}_{4}^{\prime}$ of $\mathscr{L}_{2}$ consisting of meromorphic functions on a finite ramified covering $q: W \rightarrow D \times V$ such that the extension $\mathscr{L}_{4}^{\prime} \supset \mathscr{L}_{2}$ is isomorphic to the extension $\mathscr{K}_{4}^{\prime} \supset \mathscr{K}_{2}$. Let $\mathscr{L}_{3}$ be the differential subfield of $\mathscr{L}_{4}^{\prime}$ which corresponds to $\mathscr{K}_{3}$. Since tr. d. $\left[\mathscr{L}_{3}: \mathscr{K}_{1}\right]=1$, we may assume $\mathscr{L}_{3}=\mathscr{K}_{1}\langle h\rangle$ for an appropriate element $h \in \mathscr{L}_{3}$. As $h \in \mathscr{L}_{4}^{\prime}$ is a meromorphic function on $W$, we may write $h=h\left(x_{0} ; z ; x\right)$. Since tr.d. $\left[\mathscr{L}_{3}: \mathscr{K}_{1}\right]=1, h$ satisfies a differential equation

$$
\mathscr{F}\left(x ; h, h^{\prime}\right)=0
$$

with coefficients in $\mathscr{K}_{1}$. Let $Y$ be the projective non-singular model of an algebraic curve $\left\{\left(u_{0}, u_{1}\right) \in \mathbb{C}^{2} \mid \mathscr{F}\left(x_{0} ; u_{0}, u_{1}\right)=0\right\}$ and let us put $\tilde{X}=W \cap q^{-1}(x)_{0}$. Replacing $x_{0}$ if necessary, we may assume that we have a dominant rational map $\tilde{X} \ldots Y$ and hence $h\left(x_{0} ; z ; x\right)$ is the general solution of $\mathscr{F}=0$ when $z$ varies in an open set of $X$ for the usual topology. We may put $h\left(x_{0} ; z ; x\right)=\tilde{h}\left(x_{0} ; u ; x\right)$ where $u$ varies in an open set of $Y$ for the usual topology. If we take another general point $x_{1}$, we get a function $\tilde{h}\left(x_{0} ; u\right.$; $x_{1}$ ) of $u$ defined on an open set of $Y$ for the usual topology. We know that

(62) the singularities of the function $h\left(x_{0} ; u ; x_{1}\right)$ of $u$ are at most 
algebraic branch points (see for example pp. 284-285 Leçons de Stockholm in $[\mathrm{P}])$.

Let $A_{1}\left(x_{0} ; u ; x_{1}\right)$ be the $i$-th symmetric function of the $\tilde{h}^{g}$ 's for $g \in G$. Since $A_{i}\left(x_{0} ; u ; x_{1}\right)$ is a single valued function of $u$, when the points $x_{0}, x_{1}$ are fixed, $A_{i}\left(x_{0} ; u ; x_{1}\right)$ is a meromorphic function on $Y^{\text {an }}$ by (62). The point $x_{0}$ is chosen and fixed once for all. We varying $x_{1}$ and denoting $x_{1}$ by $x, A_{i}\left(x_{0} ; u ; x\right)=\widetilde{A}_{i}(u, x)$ is a rational function of $u$ whose coefficients are holomorpihc functions of $x$ by the relative G.A.G.A. applied for the projection $p_{2}: Y \times D \rightarrow D$ (cf. [G]). We have $\tilde{h}\left(x_{0} ; u ; x\right)^{l}-\tilde{A}_{1}(u, x) \tilde{h}\left(x_{0} ; u\right.$; $x)^{l-1}+\cdots \pm \tilde{A}_{l}(u, x)=0$, where $l$ denotes the order of the Galois group G. Therefore by Lemma (19.1), the extension $\mathscr{L}_{3} \supset \mathscr{L}_{1}$ (and hence also the extension $\mathscr{K}_{3} \supset \mathscr{K}_{1}$ ) depends algebraically on the initial conditions. Namely there exists a differential field $\mathscr{M} \supset \mathscr{K}_{1}$ such that tr. d. $\left[C_{Q\left(x_{2} \otimes_{\mathscr{N}_{1}} \mathscr{\alpha}\right)}, C_{\mathscr{A}}\right]$ $=1$. Now we can argue as in subcase (60). Therefore the condition (1') implies (3).

In our paper [U2], we proved that any solution the first differential equation $y^{\prime \prime}=6 y^{2}+x$ of Painleve is not obtained from the field $\mathbb{C}$ of constants functions by a finite iteration of the permissible operations $(0)$, (P1), (P2), ‥, (P6). Among these operations, the combination of the first 6 operations (O), (P1), (P2), $\cdots,(\mathrm{P} 5)$ is equivalent to allowing $G$-primitive extensions in the language of Kolchin (cf. Definition (4)). Therefore these operations are of group theoretic nature. However in the proof of Theorem in [U2] the operation (P6) came in. Theorem (50) says that for a differential equation satisfying the conditions (44) we can do without the operation (6) whose we do not know the nature and hence the Theorem in [U2] is quite group theoretic. Therefore the irreducibility theorem in [U2] is understood in a perfect theoretic simplicity.

Let us show how to use Theorem (50) to prove the irreducibility of the differential equations of Painlevé.

Let us consider a differential equation

$$
A\left(y, y^{\prime}\right)-B\left(y, y^{\prime}\right) y^{\prime \prime}=0,
$$

where $A\left(Y, Y^{\prime}\right)$ and $B\left(Y, Y^{\prime}\right)$ are polynomials of $Y$ and $Y^{\prime}$ with coefficients in a differential field $K$ of meromorphic functions on a domain $D \subset C$. We assume that $A$ and $B$ are mutually prime in a unique factorization domain $K\left[Y, Y^{\prime}\right]$. The following condition (J) plays an improtant role in the proof of the irreducibility theorem. 
(J) For any differential field extension $L$ of $K$ if polynomials $G, H \in$ $L\left[Y, Y^{\prime}\right]$ satisfy $B\left({ }^{\prime} G\right)+B Y^{\prime} \partial G / \partial Y+A \partial G / \partial Y^{\prime}=H G$, then $G \in L\left(\subset L\left[Y, Y^{\prime}\right]\right)$, where ' $G$ is a polynomial obtained by applying the derivation of $L$ on the coefficients of $G$.

EXAMPLE (64). In the course of the proof of the irreducibility theorem in [U2], we proved that the first differential equation $y^{\prime \prime}=6 y^{2}+x$ satisfies the condition (J) (if we take $K=\mathbb{C}(x)$ ).

Corollary (65) to Theorem (50). Assume that the differential equation (47) satisfies the conditions (44) and the condition (J) over K. Let $y(x)$ be a particular solution (holomorphic over a subdomain of $D$ ) such that $B\left(y, y^{\prime}\right) \neq 0$ and tr. d. $[K\langle y(x)\rangle ; K] \geq 1$. Then (1) tr. d. $[K\langle y(x)\rangle ; K]$ $=2$ and $(2) y(x)$ is not obtained from $K$ by a finite iteration of the permissible operations $(\mathrm{O}),(\mathrm{P} 1),(\mathrm{P} 2), \cdots,(\mathrm{P} 5)$.

Proof. If tr. d. $[K\langle y(x)\rangle: K]=1$, then there would be a non-zero polynomial $E\left(Y, Y^{\prime}\right) \in K\left[Y, Y^{\prime}\right]$ such that $E\left(y, y^{\prime}\right)=0$. Since $y(x)$ is not algebraic over $K$, we have $\partial E / \partial Y^{\prime} \neq 0$ and $y(x)$ would be algebraic over $K(y)$. We choose among such $E$ a minimal polynomial $G\left(y, Y^{\prime}\right)$ of $y^{\prime}$ over $K(y)$ whose coefficients are in $K[y]$. Since $y$ is transcendental over $K$, we may further assume that the polynomial $G\left(y, Y^{\prime}\right) \in K[y]\left[Y^{\prime}\right]$ with coefficients in a unique factorization domain $K[y]$ is primitive. Differentiating $G\left(y, y^{\prime}\right)$, we would get $0={ }^{\prime} G\left(y, y^{\prime}\right)+y^{\prime} \partial G / \partial Y\left(y, y^{\prime}\right)+y^{\prime \prime} \partial G / \partial Y^{\prime}(y$, $\left.y^{\prime}\right)={ }^{\prime} G\left(y, y^{\prime}\right)+y^{\prime} \partial G / \partial Y\left(y, y^{\prime}\right)+A / B\left(y, y^{\prime}\right) \partial G / \partial Y^{\prime}\left(y, y^{\prime}\right)$. Therefore $\left(B\left({ }^{\prime} G\right)\right.$ $\left.+B Y^{\prime} \partial G / \partial Y+A \partial G / \partial Y^{\prime}\right)\left(y, y^{\prime}\right)=0$ and hence the polynomial $\left(B\left(^{\prime} G\right)+\right.$ $\left.B Y^{\prime} \partial G / \partial Y+A \partial G / \partial Y^{\prime}\right)\left(y, Y^{\prime}\right)$ would be divisible by the minimal polynomial $G\left(y, Y^{\prime}\right)$ in $K(y)\left[Y^{\prime}\right]$ : there would exist a polynomial $H\left(y, Y^{\prime}\right) \in K(y)\left[Y^{\prime}\right]$ such that

$$
\left(B\left(^{\prime} G\right)+B Y^{\prime} \partial G / \partial Y+A \partial G / \partial Y^{\prime}\right)\left(y, Y^{\prime}\right)=H\left(y, Y^{\prime}\right) G\left(y, Y^{\prime}\right) .
$$

Since $y$ is transcendental over $K$, we would get

(66) $\left(B\left(^{\prime} G\right)+B Y^{\prime} \partial G / \partial Y+A \partial G / \partial Y^{\prime}\right)\left(Y, Y^{\prime}\right)=H\left(Y, Y^{\prime}\right) G\left(Y, Y^{\prime}\right)$

in $K(Y)\left[Y^{\prime}\right]$. Since $G\left(Y, Y^{\prime}\right)$ is primitive, (66) would imply $H\left(Y, Y^{\prime}\right) \in$ $K(Y)\left[Y^{\prime}\right]$ is in $K\left[Y, Y^{\prime}\right]$ and $G$ would be in $K$. This contradicts the assumption $\partial G / \partial Y^{\prime} \neq 0$ and the first assertion is proved. To prove the second assertion we need 
LEMMA (67). If the differential equation (47) satisfies the condition (J), then the general solution $y\left(x_{0} ; z ; x\right)$ depends essentially transcendentally on the initial conditions.

Proof of the lemma. Assume that the general solution $y=y\left(x_{0} ; z ; x\right)$ depends semi-transcendentally on the initial conditions. Then there exists a differential field $M$ such that tr.d. $\left[M\left(y, y^{\prime}\right): M\right]=2$ and such that tr. d. $\left[C_{M\left(y, y^{\prime}\right)}: C_{M}\right] \geq 1$. Therefore there exists a constant $c \in M\left(y, y^{\prime}\right)$ which is transcendental over $M$. Let $c=C / E\left(y, y^{\prime}\right)$ with $0 \neq C\left(Y, Y^{\prime}\right), E\left(Y, Y^{\prime}\right)$ $\in M\left[Y, Y^{\prime}\right]$. We may assume that $C$ and $E$ are mutually prime in a unique factorization domain $M\left[Y, Y^{\prime}\right]$. Differentiating $c=C / E$, we get $(C(y, y))^{\prime} E\left(y, y^{\prime}\right)-C\left(y, y^{\prime}\right)\left(E\left(y, y^{\prime}\right)\right)^{\prime}=0$. Therefore $\left({ }^{\prime} C\left(y, y^{\prime}\right)+y^{\prime} \partial C / \partial Y\left(y, y^{\prime}\right)\right.$ $\left.+A / B\left(y, y^{\prime}\right) \partial C / \partial Y^{\prime}\left(y, y^{\prime}\right)\right) E\left(y, y^{\prime}\right)-C\left(y, y^{\prime}\right)\left({ }^{\prime} E\left(y, y^{\prime}\right)+y^{\prime} \partial E / \partial Y\left(y, y^{\prime}\right)+\right.$ $\left.A / B\left(y, y^{\prime}\right) \partial E / \partial Y^{\prime}\left(y, y^{\prime}\right)\right)=0$. Multiplying $B\left(y, y^{\prime}\right)$ we get $\left\{\left(B\left({ }^{\prime} C\right)+B Y^{\prime} \partial C / \partial Y\right.\right.$ $\left.\left.+A \partial C / \partial Y^{\prime}\right) E-C\left(B\left(^{\prime} E\right)+B Y^{\prime} \partial E / \partial Y+A \partial E / \partial Y^{\prime}\right)\right\}\left(y, y^{\prime}\right)=0$. Since $y$ and $y^{\prime}$ are algebraically independent over $M$ and since $C$ and $E$ are mutually prime, there exists a polynomial $H \in M\left[Y, Y^{\prime}\right]$ such that $B\left({ }^{\prime} C\right)+B Y^{\prime} \partial C / \partial Y$ $+A \partial C / \partial Y^{\prime}=H C$ which is an equality in the polynomial ring $M\left[Y, Y^{\prime}\right]$. Since the condition (J) is satisfied, $C\left(y, y^{\prime}\right)$ is in $M$ or $C$ is not a function of $y$ and $y^{\prime}$. The above equality shows also that there exists a polynomial $I \in M\left[Y, Y^{\prime}\right]$ such that $B\left({ }^{\prime} E\right)+B Y^{\prime} \partial E / \partial Y+A \partial E / \partial Y^{\prime}=I E$. Consequently the polynomial $E\left(y, y^{\prime}\right)$ is free from $y$ and $y^{\prime}$ and $c=C / E\left(y, y^{\prime}\right)$ is in $M$. This is a cotradiction and the general solution depends essentially transcendentally on the initial conditions.

Now we are ready to prove the irreducibility theorem.

Theorem (68). Any holomorphic solution $y(x)$ of $y^{\prime \prime}=6 y^{2}+x$ is not classical. In other words, $y(x)$ is not obtained from the field $\mathbb{C}$ of the constant functions by a finite iteration of permissible operations $(\mathrm{O}),(\mathrm{P} 1)$, (P2), ..., (P5).

Proof. As we have seen in Examples (45) and (64), for the differential equation $y^{\prime \prime}=6 y^{2}+x$, the conditions (44) and (J) are satisfied over $\mathbb{C}(x)$. It follows from Lemma 0.8 in [U2] that $y(x)$ is not algebraic over $\mathbb{C}(x)$. Thus tr. d. $[\mathbb{C}(x)\langle y(x\rangle: \mathbb{C}(x)] \geq 1$. Now Theorem follows from Corollary (65). 


\section{REFERENCES}

[B] Bourbaki, N., Algèbre commutative, Chapitres 1 à 4. Paris: Masson 1985.

[Bu] Buium, A., Differential function fields and moduli of algebraic varieties, Lect. Notes Math., 1226. Berlin Heidelberg New York: Springer 1986.

[E.G.A.] Grothendieck, A. and Dieudonné, J., Eléments de géométrie algébrique I, Grund. math. Wiss., 166. Berlin Heidelberg New York: Springer 1970.

[F] Forsyth, A. R., Theory of differential equations Part II, Ordinary equations, not linear, London: Cambridge University Press 1900.

[G] Grothendieck, A., Sur les faisceaux algébriques et les faisceaux analytiques coherents, Sém. H. Cartan, Exposé 2 (1956/57), New York Amsterdam: Benja$\min 1967$.

[I] Ince, E., Ordinary differential equations, London: Longmans 1927.

[K1] Kolchin, E., Existence Theorems connected with the Picard-Vessiot theory of homogeneous linear ordinary differential equations, Bull. Amer. Math. Soc., 54 (1948), 927-932.

[K2] —, Differential algebra and the algebraic groups, New York and London: Academic Press 1973.

[M1] Matsuda, M., Algebraic differential equation of the first order free from parametric singularities from the differential-algebraic stand point, J. Math. Soc. Jap., 30 (1978), 447-455.

[M2] - First order algebraic differential equations, Lect. Notes Math., 804, Berlin Heidelberg New York: Springer 1980.

[N1] Nishioka, K., A note on the transcendency of Painlevé's first transcendent, Nagoya Math. J., 109 (1988), 63-67.

[N2] — General solutions depending rationally on arbitrary constants, Nagoya Math. J., 113 (1989), 1-6.

[N3] - Differential algebraic function fields depending rationally on arbitrary constants, Nagoya Math. J., 113 (1989), 173-179.

[O] Okamoto, K., Sur les feuilletages associés aux équations du second ordre à points critiques fixes de P. Painlevé, Japan J. Math., 5 (1979), 1-79.

[P] Painlevé, P., Oeuvres, Paris: Edition du C. N. R. S. 1972.

[S1] Seidenberg, A., Abstract differential algebra and the analytic case, Proc. Amer. Math. Soc., 9 (1958), 159-164.

[S2] - Abstract differential algebra and the analytic case II, Proc. Amer. Math. Soc., 23 (1969), 689-691.

[U1] Umemura, H., Birational automorphism groups and differential equations, prepared for the Proc. Franco-Japanese colloquium on differential equations, Strasbourg, 1985.

[U2] - On the irreducibility of the first differential equation of Painlevé, Algebraic Geometry and Commutative Algebra in honor of Masayoshi NAGATA, Tokyo: Kinokuniya 1987, 771-789.

[W] Weil, A., The field of definition of a variety. Amer. J. Math., 78 (1956), 509-524 (= Collected papers vol. II, 291-306. Berlin Heidelberg New York: Springer 1980).

Department of Mathematics

Kumamoto University

Kumamoto, 860 Japan 